\title{
Multilocus Genotyping of Giardia duodenalis in Mostly Asymptomatic Indigenous People from the Tapirapé Tribe, Brazilian Amazon
}

\author{
Pamela Carolina Köster ${ }^{1,+}{ }^{\text {, Antonio F. Malheiros }}{ }^{2,3,+}{ }^{\mathbb{D}}$, Jeffrey J. Shaw ${ }^{4}$, Sooria Balasegaram ${ }^{5}$, \\ Alexander Prendergast ${ }^{6}$, Héloïse Lucaccioni ${ }^{7}$ (D), Luciana Melhorança Moreira ${ }^{3}$, Larissa M. S. Lemos ${ }^{8}$, \\ Alejandro Dashti ${ }^{1}$, Begoña Bailo ${ }^{1}$, Arlei Marcili ${ }^{9,10}$, Herbert Sousa Soares ${ }^{9}$, Solange Maria Gennari ${ }^{9,10} \mathbb{D}^{\text {, }}$ \\ Rafael Calero-Bernal ${ }^{11}$, David González-Barrio ${ }^{1}$ and David Carmena ${ }^{1, *(D)}$
}

1 Parasitology Reference and Research Laboratory, Spanish National Centre for Microbiology, Majadahonda, 28220 Madrid, Spain; pamelakster@yahoo.com (P.C.K.); dashti.alejandro@gmail.com (A.D.); BEGOBB@isciii.es (B.B.); david.gonzalezb@isciii.es (D.G.-B.)

2 Post-Graduation Program in Environmental Science, Faculty of Agricultural and Biological Sciences, University of State of Mato Grosso, Cáceres, MG 78200-000, Brazil; malheiros@unemat.br

3 Department of Biological Sciences, Faculty of Agricultural and Biological Sciences, University of State of Mato Grosso, Cáceres, MG 78200-000, Brazil; luciana.melhoranca@unemat.br

4 Department of Parasitology, Institute of Biomedical Sciences, São Paulo University, São Paulo, SP 05508-000, Brazil; jayusp@hotmail.com

check for updates

Citation: Köster, P.C.;

Malheiros, A.F.; Shaw, J.J.; Balasegaram, S.; Prendergast, A.; Lucaccioni, H.; Moreira, L.M.; Lemos, L.M.S.; Dashti, A.; Bailo, B.; et al. Multilocus Genotyping of Giardia duodenalis in Mostly Asymptomatic Indigenous People from the Tapirapé Tribe, Brazilian Amazon. Pathogens 2021, 10, 206. https://doi.org/10.3390/ pathogens10020206

Academic Editor: Siddhartha Das

Received: 14 December 2020

Accepted: 8 February 2021

Published: 14 February 2021

Publisher's Note: MDPI stays neutral with regard to jurisdictional claims in published maps and institutional affiliations.

Copyright: (c) 2021 by the authors. Licensee MDPI, Basel, Switzerland. This article is an open access article distributed under the terms and conditions of the Creative Commons Attribution (CC BY) license (https:/ / creativecommons.org/licenses/by/ $4.0 /)$.
5 Field Epidemiology Services, National Infection Service, Public Health England, London SE1 8UG, UK; Sooria.Balasegaram@phe.gov.uk

6 Independent Researcher, Croydon CR0, UK; akprendergast4@gmail.com

7 European Program for Intervention Epidemiology Training (EPIET), European Centre for Disease Prevention and Control (ECDC), 16973 Stockholm, Sweden; heloiselucaccioni@dgs.min-saude.pt

8 Department of Nursing, Faculty of Health Sciences, University of State of Mato Grosso, Cáceres, MG 78200-000, Brazil; larissa@unemat.br

9 Post-Graduation Program in Veterinary Medicine, Santo Amaro University, São Paulo, SP 04829-300, Brazil; amarcili@usp.br (A.M.); hesoares@prof.unisa.br (H.S.S.); sgennari@usp.br (S.M.G.)

10 Department of Preventive Veterinary Medicine and Animal Health, School of Veterinary Medicine, University of São Paulo, São Paulo, SP 05508-270, Brazil

11 SALUVET Research Group, Department of Animal Health, Faculty of Veterinary, Complutense University of Madrid, 28040 Madrid, Spain; r.calero@ucm.es

* Correspondence: dacarmena@isciii.es; Tel.: +34-91-822-3641

+ These two authors contributed equally to this work.

Abstract: Little information is available on the occurrence and genetic variability of the diarrhoeacausing enteric protozoan parasite Giardia duodenalis in indigenous communities in Brazil. This cross-sectional epidemiological survey describes the frequency, genotypes, and risk associations for this pathogen in Tapirapé people (Brazilian Amazon) at four sampling campaigns during 2008-2009. Microscopy was used as a screening test, and molecular (PCR and Sanger sequencing) assays targeting the small subunit ribosomal RNA, the glutamate dehydrogenase, the beta-giardin, and the triosephosphate isomerase genes as confirmatory/genotyping methods. Associations between G. duodenalis and sociodemographic and clinical variables were investigated using Chi-squared test and univariable/multivariable logistic regression models. Overall, 574 individuals belonging to six tribes participated in the study, with G. duodenalis prevalence rates varying from $13.5-21.7 \%$. The infection was positively linked to younger age and tribe. Infected children $<15$ years old reported more frequent gastrointestinal symptoms compared to adults. Assemblage B accounted for three out of four G. duodenalis infections and showed a high genetic diversity. No association between assemblage and age or occurrence of diarrhoea was demonstrated. These data indicate that the most likely source of infection was anthropic and that different pathways (e.g., drinking water) may be involved in the transmission of the parasite.

Keywords: Giardia; Brazil; Amazon; asymptomatic; community; genotyping; indigenous; risk association; Tapirapé; transmission 


\section{Introduction}

The flagellated Giardia duodenalis (syn. G. intestinalis, G. lamblia) is a cosmopolitan protozoan parasite that inhabits the gastrointestinal tract of humans and other vertebrate animals. Giardiasis is the most reported intestinal protozoan infection globally, with an estimated 280 million symptomatic cases every year [1]. Asymptomatic infections are even more frequent, both in developing [2,3] and developed [4] countries. Indeed, large epidemiological case-control studies conducted in high-prevalence settings have demonstrated that $G$. duodenalis infection was significantly more common in asymptomatic controls than in cases with diarrhoea [5-7]. Host immune status and level of nutrition seem to be key factors in the control of the infection or its progression to active disease [8], although the genotype of the parasite may also play a role in the health/disease balance of the host [9]. When present, clinical manifestations associated with G. duodenalis infection may include self-limiting acute diarrhoea, persistent diarrhoea, epigastric pain, nausea, and vomiting [10]. Long-term sequelae, including childhood growth retardation and cognitive impairment, have also been recognised [11,12]. Contrary to severe infections by other diarrhoea-causing protozoan parasites such as Cryptosporidium spp. or Entamoeba histolytica, giardiasis is rarely fatal and is better considered as a debilitating condition.

Transmission of G. duodenalis is through the faecal-oral route, either directly via direct contact with infected humans or animals, or indirectly via ingestion of contaminated food or water. Waterborne transmission is likely the most common source of human infections in poor-resource settings with little or no access to safe drinking water and insufficient sanitary facilities [3]. Because of its strong bond with poverty and elevated socioeconomic impact, giardiasis (together with cryptosporidiosis) joined the Neglected Diseases Initiative launched by the World Health Organisation in 2004 [13].

Giardia duodenalis exhibits a considerable degree of genetic heterogeneity, allowing the differentiation of eight $(\mathrm{A}-\mathrm{H})$ lineages or assemblages with marked differences in host specificity and range [14]. These genetic variants likely represent cryptic species [15]. Assemblages A and B cause most human infections, but they can also infect other mammalian hosts and are, therefore, considered potentially zoonotic. Assemblages $\mathrm{C}$ and $\mathrm{D}$ occur mainly in canids, assemblage $\mathrm{E}$ in domestic and wild ungulates, assemblage $\mathrm{F}$ in cats, assemblage $\mathrm{G}$ in rodents, and assemblage $\mathrm{H}$ in marine pinnipeds. Human infections by assemblages $\mathrm{C}-\mathrm{F}$ have been sporadically reported, particularly in children and immunocompromised individuals [14].

A recent review on the epidemiological situation of G. duodenalis in Brazil has revealed that this protozoan parasite represents a public health concern in the country, with prevalence rates up to 78\% in Minas Gerais State and 70\% in São Paulo State in 1998 [16]. Available molecular data in the country have evidenced marked differences in the geographical segregation of $G$. duodenalis assemblages circulating in human populations (Table S1), domestic and wildlife animal species (Table S2), surface waters (Table S3), and fresh produce (Table S4), likely reflecting disparities in infection sources and transmission pathways. Indeed, contaminated surface waters and having contact with domestic (mainly dog) animals were considered as probable sources of human infections [16]. Despite this relative abundance of epidemiological data, giardiasis has been poorly studied in Brazilian indigenous people, partially due to the geographical isolation and difficulty in accessing these fragile communities. Thus, G. duodenalis infections have been documented by conventional (microscopy) methods in the range of $7-47 \%$ in the Parakanã indigenous people in the eastern Amazon region [17], in indigenous communities in the municipality of São Gabriel da Cachoeira, Amazonas State [18], in native Brazilian children in the Xingu Indian Reservation, Mato Grosso State [19], in the Maxakali and Xukuru-Kariri indigenous communities, Minas Gerais State [20,21], and in the Terena indigenous people, Mato Grosso do Sul State [22]. However, no information is currently available on the G. duodenalis assemblages and sub-assemblages circulating in native Brazilian people. This 
molecular-based epidemiological survey aims at investigating the genetic diversity of G. duodenalis and assessing potential risk and/or protective factors associated with the infection in indigenous people from the Tapirapé tribe living in the Brazilian Amazon.

\section{Results}

\subsection{Study Population}

In this study, a total of 574 individuals (male/female ratio: 0.96; age range: 0.1-88 years old, median: 14.0 years old) of the Tapirapé ethnicity living in six independent tribes (population range: 40-263 inhabitants, standard deviation: 83.3) were censed and invited to participate in four consecutive sampling campaigns during July 2008 and January 2010, in both dry and wet seasons. Overall, 98\% (564/574) of the censed individuals participated at least in one sampling campaign. Participation rates ranged from $40 \%$ to $93 \%$ depending on the tribe and sampling campaign (Table 1). A total of 141 individuals participated in all four sampling campaigns, 201 in three sampling campaigns, 136 in two sampling campaigns, and 86 in a single sampling campaign. The distribution of the participating individuals according to sex, age group, and tribe of origin is also summarised in Table 1. Females (mean: 53.6\%, SD: 1.0) participated in the survey more often than males (mean: $46.4 \%$, SD: 1.0). Adults ( $>15$ years old) were the largest group in the surveyed population (mean: $24.6 \%$, SD: 3.2 ), with children 5 to 14 years of age (mean: $39.4 \%$, SD: 1.9 ) and children $\leq 5$ years old accounting, in average, for $14.6 \%$ (SD: 2.0 ) of the investigated individuals.

Table 1. Participation rates and distribution by sex, age group, and tribe of origin of the Tapirapé people $(n=564)$ taking part in the four sampling campaigns conducted in the present survey, Brazilian Amazon.

\begin{tabular}{|c|c|c|c|c|c|c|c|c|c|c|c|c|c|c|c|}
\hline \multirow[b]{2}{*}{$\begin{array}{l}\text { Sampling } \\
\text { Campaign }\end{array}$} & \multicolumn{2}{|c|}{ Participation } & \multicolumn{2}{|c|}{ Sex } & \multicolumn{5}{|c|}{ Age group (years) } & \multicolumn{6}{|c|}{ Tribe $n(\%)$} \\
\hline & Participants $(n)$ & Rate (\%) & Male & Female & $0-4$ & $5-9$ & $10-14$ & $\geq 15$ & Unknown & 1 & 2 & 3 & 4 & 5 & 6 \\
\hline $2008^{1}$ & 362 & 64 & 171 & 191 & 45 & 73 & 74 & 170 & 0 & 175 (66.5) & $31(40.3)$ & $46(65.7)$ & $47(63.5)$ & $28(56.0)$ & $35(87.5)$ \\
\hline $2009^{2}$ & 374 & 66 & 175 & 199 & 53 & 75 & 66 & 178 & 2 & $174(66.2)$ & $45(58.4)$ & $31(44.3)$ & $41(55.4)$ & $46(92.0)$ & $37(92.5)$ \\
\hline $2009^{1}$ & 407 & 72 & 189 & 218 & 59 & 90 & 64 & 192 & 2 & $202(76.8)$ & $49(63.6)$ & $36(51.4)$ & $42(56.8)$ & $45(90.0)$ & $33(82.5)$ \\
\hline $2010^{2}$ & 382 & 68 & 172 & 210 & 66 & 94 & 64 & 156 & 2 & $193(73.4)$ & $51(66.2)$ & $38(54.3)$ & $35(47.3)$ & $39(78.0)$ & $26(65.0)$ \\
\hline
\end{tabular}

\subsection{Prevalence of G. duodenalis}

Microscopy-based prevalence rates for G. duodenalis in the Tapirapé community varied from $13.5 \%$ (55/407) in the dry season of 2009 to $21.7 \%(83 / 382)$ in the rainy season of 2010 (Table 2). Over the four sampling campaigns, 35.1\% (198/564) individuals tested positive at least once. The occurrence of the parasite was influenced by the seasonality ( $22 \%$ rainy versus $17 \%$ dry season, Chi-squared test $p=0.022$ ) but not the sampling period (year) (Chi-squared test $p=0.126)$. Subsequent newly diagnosed infections were also more likely to occur in the rainy season (odds ratio: $2.29,95 \%$ CI 1.46-3.68, $p=0.0001$ ) although this is dependent on the number of samples analysed. G. duodenalis infections were more commonly identified in children aged 0-4 years old. During the period of study, G. duodenalis prevalence varied greatly within and among the six tribes investigated, but tribe 5 presented the highest infection rates in all sampling campaigns.

A total of 43,17, and 4 individuals tested positive for $G$. duodenalis in two, three, or all four sampling campaigns, respectively (Table 3), although this was dependent on the number of samples. In all cases, children younger than 15 years of age accounted for $50.0 \%$ to $88.2 \%$ of the subjects where the parasite was detected in two or more sampling campaigns. When considering observations with repeated samples, $61.7 \%$ observations were always negative, $4.2 \%$ always positive, and $34.1 \%$ discontinuously positive. Repeated G. duodenalis infections were more frequently detected in the wet season (odds ratio: 1.60, 95\% CI 1.12-2.29, $p=0.0075$ ) in members of tribe 1 (range: 50.0-58.1\%) and, to a lesser extent, in tribe 5 (range: $18.6-50.0 \%$ ). 
Table 2. Microscopy-based prevalence of Giardia duodenalis by sex, age group, and tribe of origin of the Tapirapé people $(n=564)$ participating in the present survey according to the sampling period, Brazilian Amazon.

\begin{tabular}{|c|c|c|c|c|c|c|c|c|}
\hline \multirow[b]{2}{*}{ Variable } & \multicolumn{2}{|l|}{$2008^{1}$} & \multicolumn{2}{|l|}{$2009^{2}$} & \multicolumn{2}{|l|}{$2009^{1}$} & \multicolumn{2}{|c|}{$2010^{2}$} \\
\hline & Giardia Positive & $\%$ & Giardia Positive & $\%$ & Giardia Positive & $\%$ & Giardia Positive & $\%$ \\
\hline \multicolumn{9}{|l|}{ Sex } \\
\hline Male & 31 & 19 & 35 & 20 & 30 & 16 & 39 & 23 \\
\hline Female & 37 & 18 & 46 & 23 & 25 & 12 & 43 & 21 \\
\hline \multicolumn{9}{|c|}{ Age group (years) } \\
\hline $0-4$ & 13 & 29 & 17 & 32 & 17 & 29 & 28 & 42 \\
\hline $5-9$ & 17 & 23 & 20 & 27 & 15 & 17 & 25 & 27 \\
\hline $10-14$ & 16 & 22 & 14 & 21 & 9 & 14 & 11 & 17 \\
\hline$\geq 15$ & 22 & 13 & 30 & 17 & 14 & 7 & 19 & 12 \\
\hline \multicolumn{9}{|l|}{ Tribe } \\
\hline 1 & 41 & 23 & 34 & 20 & 30 & 15 & 48 & 25 \\
\hline 2 & 2 & 7 & 10 & 22 & 7 & 14 & 6 & 12 \\
\hline 3 & 4 & 9 & 3 & 10 & 2 & 6 & 6 & 16 \\
\hline 4 & 4 & 9 & 2 & 5 & 1 & 2 & 5 & 14 \\
\hline 5 & 10 & 36 & 21 & 46 & 13 & 29 & 14 & 36 \\
\hline 6 & 7 & 20 & 11 & 30 & 2 & 6 & 4 & 15 \\
\hline Total & 68 & 19 & 81 & 22 & 55 & 14 & 83 & 22 \\
\hline
\end{tabular}

${ }^{1}$ Dry season. ${ }^{2}$ Rainy season.

Table 3. Number of individuals with a positive result to Giardia duodenalis by microscopy in two or more of the sampling campaigns conducted in the present study, Brazilian Amazon.

\begin{tabular}{|c|c|c|c|c|c|c|}
\hline Variable & $\begin{array}{c}\text { Positives in } 2 \\
\text { Campaigns ( }(n)\end{array}$ & Frequency (\%) & $\begin{array}{l}\text { Positives in } 3 \\
\text { Campaigns }(n)\end{array}$ & Frequency (\%) & $\begin{array}{l}\text { Positives in all } 4 \\
\text { Campaigns }(n)\end{array}$ & Frequency (\%) \\
\hline \multicolumn{7}{|c|}{ Age group (years) } \\
\hline $0-4$ & 14 & 33 & 6 & 35 & 1 & 25 \\
\hline $5-9$ & 12 & 28 & 6 & 35 & 1 & 25 \\
\hline $10-14$ & 9 & 21 & 3 & 18 & 0 & 0 \\
\hline$\geq 15$ & 8 & 19 & 2 & 12 & 2 & 50 \\
\hline Total & 43 & 100 & 17 & 100 & 4 & 100 \\
\hline \multicolumn{7}{|l|}{ Tribe } \\
\hline 1 & 25 & 58 & 7 & 41 & 2 & 50 \\
\hline 2 & 4 & 9 & 1 & 6 & 0 & 0 \\
\hline 3 & 1 & 2 & 0 & 0 & 0 & 0 \\
\hline 4 & 1 & 2 & 0 & 0 & 0 & 0 \\
\hline 5 & 8 & 19 & 7 & 41 & 2 & 50 \\
\hline 6 & 4 & 9 & 2 & 12 & 0 & 0 \\
\hline Total & 43 & 100 & 17 & 100 & 4 & 100 \\
\hline
\end{tabular}

\subsection{Molecular Characterisation of G. duodenalis}

The genetic diversity within G. duodenalis was investigated in a subset of 70 stool samples from 65 individuals with a positive result for this parasite by conventional microscopy. Five individuals provided stool samples positive to this parasite at two different sampling periods. The presence of the parasite was confirmed by qPCR in 97\% (68/70) of these samples. Generated cycle threshold (Ct) values ranged from 18.2 to 35.4 (median: 27.4; SD: 3.7).

Genotyping/sub-genotyping data were obtained for a total of 63 stool samples belonging to 58 individuals (Table 4). Amplification success rates were 100\% (63/63) for glutamate dehydrogenase $(g d h)$, and 87\% (55/63) for beta-giardin $(b g)$ and triosephosphate isomerase (tpi), respectively. Multilocus sequence typing (MLST) data at the three loci were obtained from $83 \%$ (52/63) of the samples. Sequence analyses revealed the presence of assemblages 
A $(25 \% ; 16 / 63)$ and B $(68 \% ; 43 / 63)$. Mixed infections A + B were identified in $6 \%(4 / 63)$ of the samples analysed. No mixed infections involving host-specific assemblages $\mathrm{C}-\mathrm{H}$ were detected.

Table 4. Multilocus genotyping results of the 63 G. duodenalis-positive samples of human origin successfully genotyped at any of the three loci investigated in the present survey.

\begin{tabular}{|c|c|c|c|c|c|}
\hline Sample ID & Ct value in $\mathrm{qPCR}$ & $g d h$ & $b g$ & $t p i$ & Assigned Genotype \\
\hline 5 & 27.4 & BIII & $\mathrm{B}$ & BIII & BIII \\
\hline 13 & 25.1 & AII & $\mathrm{AII}+\mathrm{AIII}$ & AII & $\mathrm{AII}+\mathrm{AIII}$ \\
\hline 24 & 27.2 & AII & $\mathrm{AII}+\mathrm{AIII}$ & AII & $\mathrm{AII}+\mathrm{AIII}$ \\
\hline 31 & 26.4 & BIII/BIV & B & BIII/BIV & BIII/BIV \\
\hline 33 & 28.2 & $\mathrm{BIII} / \mathrm{BIV}$ & B & $\mathrm{BIII} / \mathrm{BIV}$ & $\mathrm{BIII} / \mathrm{BIV}$ \\
\hline 41 & 24.0 & BIII & B & $\mathrm{BIII} / \mathrm{BIV}$ & $\mathrm{BIII} / \mathrm{BIV}$ \\
\hline 49 & 25.5 & BIII/BIV & B & BIII & $\mathrm{BIII} / \mathrm{BIV}$ \\
\hline $50 a$ & 21.2 & AII & AIII & AII & AII/AIII \\
\hline $50 \mathrm{~b}$ & 23.8 & BIII & $\mathrm{AIII}+\mathrm{B}$ & BIII & $\mathrm{AIII}+\mathrm{BIII}$ \\
\hline 55 & 27.3 & BIII & B & BIV & BIII/BIV \\
\hline 58 & 29.6 & BIII & B & BIII/BIV & $\mathrm{BIII} / \mathrm{BIV}$ \\
\hline 60 & 29.1 & BIII & B & BIII/BIV & $\mathrm{BIII} / \mathrm{BIV}$ \\
\hline 70 & 34.0 & BIII & - & - & BIII \\
\hline 71 & 30.0 & BIII & B & BIII/BIV & BIII/BIV \\
\hline 72 & 21.7 & BIII & B & $\mathrm{BIII} / \mathrm{BIV}$ & BIII/BIV \\
\hline 78 & 18.2 & BIII & B & BIII & BIII \\
\hline 79 & 29.4 & BIII/BIV & B & BIII & BIII/BIV \\
\hline 82 & 30.5 & BIII & B & $\mathrm{AII}+\mathrm{BIII}$ & $\mathrm{AII}+\mathrm{BIII}$ \\
\hline 85 & 29.7 & BIII/BIV & B & BIII & BIII/BIV \\
\hline $86 a$ & 27.0 & BIII & B & BIII/BIV & BIII/BIV \\
\hline $86 b$ & 26.3 & BIII & B & BIII/BIV & BIII/BIV \\
\hline $93 a$ & 30.4 & BIII/BIV & B & BIII/BIV & BIII/BIV \\
\hline $93 b$ & 24.9 & BIII/BIV & B & BIII/BIV & BIII/BIV \\
\hline 94 & 22.0 & AII & - & AII & AII \\
\hline 106 & 21.3 & AII & AIII & AII & AII/AIII \\
\hline 111 & 26.2 & AII & AIII & AII & AII/AIII \\
\hline 123 & 34.9 & BIII & - & - & BIII \\
\hline 128 & 30.5 & AII & AIII & AII & AII/AIII \\
\hline 131 & 23.5 & BIII/BIV & B & BIII/BIV & BIII/BIV \\
\hline 132 & 29.5 & BIII & B & BIII & BIII \\
\hline 149 & 23.6 & BIII & B & - & BIII \\
\hline 157 & 28.2 & BIII/BIV & B & BIII & BIII/BIV \\
\hline 168 & 24.0 & BIII/BIV & B & BIII & BIII/BIV \\
\hline 172 & 28.3 & BIII/BIV & B & BIII & BIII/BIV \\
\hline $176 \mathrm{~b}$ & 29.3 & BIII & - & - & BIII \\
\hline 179 & 27.5 & AII & B & AII & $\mathrm{AII}+\mathrm{B}$ \\
\hline 180 & 23.3 & BIII/BIV & B & BIII/BIV & BIII/BIV \\
\hline 182 & 27.5 & BIII/BIV & B & BIII & BIII/BIV \\
\hline 184 & 31.3 & AII & - & AII & AII \\
\hline 186 & 26.3 & BIII/BIV & B & BIII & BIII/BIV \\
\hline 198 & 22.6 & BIII/BIV & B & BIII & BIII/BIV \\
\hline 200 & 25.2 & BIII/BIV & B & BIII & BIII/BIV \\
\hline 216 & 25.8 & BIII & B & - & BIII \\
\hline 227 & 19.5 & AII & $\mathrm{AII}+\mathrm{AIII}$ & AII & $\mathrm{AII}+\mathrm{AIII}$ \\
\hline 242 & 28.3 & BIII/BIV & B & BIII & BIII/BIV \\
\hline 245 & 22.4 & BIII & B & BIII & BIII \\
\hline $269 a$ & 31.2 & BIII & - & BIII & BIII \\
\hline $269 b$ & 25.1 & AII & AII & AII & AII \\
\hline $282 a$ & 27.3 & BIII/BIV & B & BIII & BIII/BIV \\
\hline $282 b$ & 23.4 & BIII/BIV & B & BIII & BIII/BIV \\
\hline 328 & 32.0 & AII & - & - & AII \\
\hline 330 & 30.3 & BIII/BIV & B & - & BIII/BIV \\
\hline 353 & 25.3 & BIII & B & BIII & BIII \\
\hline 374 & 21.2 & BIII/BIV & B & BIII & BIII/BIV \\
\hline 379 & 23.7 & AII & $\mathrm{AII}+\mathrm{AIII}$ & AII & $\mathrm{AII}+\mathrm{AIII}$ \\
\hline 420 & 19.2 & AII & $\mathrm{AII}+\mathrm{AIII}$ & AII & $\mathrm{AII}+\mathrm{AIII}$ \\
\hline 570 & 24.7 & BIII/BIV & B & BIII & BIII/BIV \\
\hline 572 & 28.3 & AII & $\mathrm{AII}+\mathrm{B}$ & AII & $\mathrm{AII}+\mathrm{B}$ \\
\hline 585 & 24.9 & BIII & B & BIII & BIII \\
\hline 595 & 25.1 & AII & AIII & - & AII/AIII \\
\hline 596 & 35.4 & AII & - & - & AII \\
\hline 604 & 21.7 & BIII & B & BIII/BIV & BIII/BIV \\
\hline 607 & 28.1 & $\mathrm{BIII}$ & B & BIII & BIII \\
\hline
\end{tabular}

bg, beta-giardin; Ct, cycle threshold; gdh, glutamate dehydrogenase; qPCR: real-time polymerase chain reaction; tpi, triosephosphate isomerase.

Subtyping analyses revealed that sub-assemblage AII $(8 \%, 5 / 63)$, mixed AII + AIII infections $(8 \%, 5 / 63)$, and ambiguous AII/AIII results $(8 \%, 5 / 63)$ were equally distributed within assemblage A. No isolates were identified as sub-assemblage AIII. Within assemblage $B$, most $(50 \%, 31 / 63)$ of the sequences corresponded to ambiguous BIII/BIV results. 
BIII was identified in $21 \%(13 / 63)$ of the sequences, whereas no isolates belonging to BIV were detected. Out of the four A + B mixed infections detected, one $(2 \%, 1 / 63)$ involved sub-assemblages AII + BIII, one $(2 \%, 1 / 63)$ sub-assemblages AIII + BIII, and two ( $3 \%$, 2/63) sub-assemblage AII + B (unknown sub-assemblage). Out of the five individuals with giardiasis at two consecutive sampling periods, three of them (ID: 86, 93, and 282) were infected by BIII/BIV at both sampling periods, indicative of prolonged G. duodenalis infection, or re-infection by that very same genotype. In contrast, one individual (ID: 50) was first infected by AII/AIII, and by AIII + B at the following sampling campaign. The remaining individual (ID: 269) was first infected by BIII and then by AII at the following sampling campaign. Both cases were strongly suggestive of re-infection events by different genotypes of the parasite.

\subsection{Intra-Assemblage Genetic Diversity}

Tables 5-7, Table S5 show the genetic diversity of the $g d h, b g$, and tpi representative, partial sequences generated in the present study. These Tables provide information for each sequence including stretch, single nucleotide polymorphisms (SNPs), and GenBank accession number. Assemblage/sub-assemblage assignment was conducted by direct comparison of the sequencing results obtained at the three loci investigated. Sequences presenting double peak positions that could not be unequivocally assigned to a given assemblage/sub-assemblage were reported as ambiguous sequences.

A total of 63 sequences were successfully characterised at the $g d h$ locus (Table 5). All 17 assemblage A sequences were unequivocally identified as sub-assemblage AII. Of them, seven sequences were $100 \%$ identical to reference sequence L40510. The remaining 10 sequences differed by 1-6 SNPs from L40510. BIII sequences showed a high degree of genetic diversity among them, explaining that $21 / 24$ of the sequences assigned to this sub-assemblage corresponded to distinct genotypes (genetic variants) of the parasite. These sequences differed by 4-13 SNPs from reference sequence AF069059, most of them associated with ambiguous (double peak) positions. Similarly, most (20/22) sequences identified as ambiguous BIII/BIV sequences were different among them, differing by 9-17 SNPs from reference sequence L40508. Virtually all SNPs detected in BIII/IV sequences corresponded to double peaks at single nucleotide positions.

At the $b g$ locus, a total of 55 sequences were fully characterised (Table 6). Out of the 14 assemblage A sequences, two belonged to AII and five to AIII. All AII and AIII sequences were identical to reference sequences AY072723 and AY072724, respectively. Five sequences were considered mixed AII + AIII infections based on the presence of two double peak (C415Y and T423Y) positions and taking sequence AY072723 as reference. Two additional sequences corresponded to AII + B and AIII + B mixed infections, differing by 32 and 38 SNPs from reference sequence AY072727, respectively. Except one, all the detected SNPs corresponded to clear double peak positions. Compared to the $g d h$ locus, a lower (but still substantial) degree of genetic variability was observed within the 41 sequences assigned to assemblage B at the $b g$ locus. All of them differed by 1-6 SNPs from reference sequence AY072727. A genetic variant showing two transitional mutations at positions $\mathrm{C} 165 \mathrm{~T}$ and $\mathrm{A} 183 \mathrm{G}$ was the genotype most frequently detected.

A total of 55 sequences were fully characterised at the tpi locus (Table 7). Within assemblage A, 14 sequences were assigned to the sub-assemblage AII. Of them, eight had $100 \%$ homology with reference sequence U57897, whereas the remaining six sequences differed by 1-2 SNPs from the latter. Two additional sequences were identified as AII + BIII sequences and presented 94-95 SNPs when aligned with reference sequence U57897. Out of the 25 sequences assigned to BIII, seven showed 100\% identity with reference sequence AF069561. The remaining 18 sequences grouped in 16 distinct genotypes that differed by $1-6$ SNPs from reference sequence A AF069561. Only a single sequence was confirmed ad BIV, differing by 3 SNPs with reference sequence AF069560. Finally, virtually all (12/13) sequences with a BIII/BIV ambiguous result were different among them, differing from reference sequence AF069560 by 7-11 SNPs. As in the case of the BIII/BIV sequences identified at the $g d h$ locus, most of the SNPs identified at the tpi locus were associated with ambiguous nucleotide positions. 
Table 5. Diversity, frequency, and main molecular features of G. duodenalis sequences at the $g d h$ locus generated in the present study. GenBank accession numbers are provided.

\begin{tabular}{|c|c|c|c|c|c|c|}
\hline Assemblage & Sub-Assemblage & No. of Isolates & Reference Sequence & Stretch & Single Nucleotide Polymorphisms & GenBank ID \\
\hline \multirow[t]{7}{*}{ A } & AII & 7 & L40510 & 64-491 & None & MT542718 \\
\hline & & 1 & L40510 & $66-486$ & T88C, C128T, C179T, T385C, A410G, T418A & MT542719 \\
\hline & & 1 & L40510 & 64-491 & T132Y, C179T, A224M, G332R & MT542720 \\
\hline & & 1 & L40510 & 64-491 & C150Y, C179T & MT542721 \\
\hline & & 6 & L40510 & $64-491$ & $\mathrm{C} 179 \mathrm{~T}$ & MT542722 \\
\hline & & 1 & L40510 & $68-491$ & C179T, T355Y & MT542723 \\
\hline & & 1 & L40510 & $64-491$ & C179Y & MT542724 \\
\hline \multirow[t]{36}{*}{ B } & BIII & 1 & AF069059 & $28-455$ & C39T, C87Y, C99Y, C123Y, T147Y, G150R, C204Y, C309Y & MT542725 \\
\hline & & 1 & AF069059 & $30-455$ & C39T, T219Y, C309T, C351Y & MT542726 \\
\hline & & 1 & AF069059 & $28-455$ & C39T, C87Y, C135Y, T147Y, G150R, C204Y, C309T, C336Y & MT542727 \\
\hline & & 1 & AF069059 & $28-455$ & C39Y, C99Y, T147Y, G150R, C204Y, C360Y, C387Y, G402R & MT542728 \\
\hline & & 2 & AF069059 & $40-455$ & C87Y, C99Y, C123Y, T147Y, G150R, C204Y, C309T & MT542730 \\
\hline & & 1 & AF069059 & $40-455$ & C87Y, C99Y, C123Y, T147Y, G150R, C204Y, C309T, G402R & MT542731 \\
\hline & & 1 & AF069059 & $47-455$ & C87Y, C99Y, T147Y, G150R, C204Y, T237Y, C309Y, G354R, G372R, C375Y & MT542732 \\
\hline & & 1 & AF069059 & $47-455$ & C87Y, C132Y, T147Y, G150R, C174Y, C204Y, T237Y, C309Y, A324R, G354R, G372R, C375Y, G402R & MT542733 \\
\hline & & 2 & AF069059 & $49-455$ & C87Y, C132Y, T147Y, G150R, C174Y, C204Y, C309Y, A324R, G372R, C375Y, G402R & MT542734 \\
\hline & & 1 & AF069059 & $41-460$ & C87T, C132T, T147C, G150A, C174T, C204T, A324G, G402A & MT542735 \\
\hline & & 1 & AF069059 & $40-455$ & C87T, C132Y, T147C, G150A, C174T, C204T, A324R, G402A & MT542736 \\
\hline & & 1 & AF069059 & $44-443$ & C87T, C132T, T147C, G150A, C174Y, C204T, G402A, G406A & MT542737 \\
\hline & & 1 & AF069059 & $46-454$ & C87Y, C135Y, T147Y, G150R, T209Y, C309T, C336Y & MT542738 \\
\hline & & 1 & AF069059 & $40-455$ & C87Y, T147Y, G150R, T237Y, C309Y, G354R, C360Y, C387Y, G402R & MT542739 \\
\hline & & 2 & AF069059 & $40-455$ & C87Y, C204Y, C309T, G372R, C375Y & MT542740 \\
\hline & & 1 & AF069059 & $48-455$ & C99Y, C135Y, T147Y, G150R, C204Y, C309Y & MT542741 \\
\hline & & 1 & AF069059 & $82-443$ & C99T, T147C, G150A, C204T & MT542742 \\
\hline & & 1 & AF069059 & $46-455$ & C87Y, C99Y, C123Y, C132Y, T147Y, G150R, C174Y, C204Y, C309Y, A324R, G402R & MT542744 \\
\hline & & 1 & AF069059 & $40-455$ & C87T, T147C, G150A, C309T & MT542745 \\
\hline & BIII/BIV & 1 & L40508 & $76-491$ & G84R, C159Y, T183Y, G186R, C255Y, C273Y, C345Y, T366Y, T387C, G408R, A438G & MT542746 \\
\hline & & 1 & L40508 & $85-491$ & C123Y, T135Y, C159Y, T183Y, G186R, C240Y, C255Y, C273Y, C345Y, T366Y, T387Y, A438R & MT542747 \\
\hline & & 2 & L40508 & $76-491$ & C123Y, T135Y, C168Y, G180R, T183Y, G186R, C210Y, C240Y, C255Y, C273Y, A360R, T366Y, T387Y & MT542748 \\
\hline & & 1 & L40508 & $76-491$ & $\begin{array}{c}\text { C123Y, T135Y, C168Y, T183Y, G186R, C210Y, C240Y, C255Y, C273Y, C291Y, C345Y, A360R, T366Y, } \\
\text { C372Y, T387Y, C423Y, A438R }\end{array}$ & MT542749 \\
\hline & & 1 & L40508 & 76-491 & $\begin{array}{l}\text { C123Y, T135Y, C168Y, T183Y, G186R, C210Y, C240Y, C255Y, C273Y, C345Y, A360R, T366Y, C372Y, } \\
\text { T387Y C423Y, A438R }\end{array}$ & MT542750 \\
\hline & & 1 & L40508 & $101-491$ & C123Y, T135Y, C168Y, T183Y, G186R, C210Y, C240Y, C255Y, C273Y, C345Y, T366C, T387Y, A438R & MT542751 \\
\hline & & 2 & L40508 & $77-491$ & C123Y, T135Y, C171Y, G180R, C240Y, C255Y, C273Y, C345Y, T366Y, T387Y, C423Y, A438R & MT542752 \\
\hline & & 1 & L40508 & $76-491$ & C123Y, T135Y, T183Y, C240Y, C255Y, C273Y, C291Y, C345Y, T366Y, C372Y, T387Y, G408R, C411Y, A438R & MT542753 \\
\hline & & 1 & L40508 & 76-491 & C123Y, T135Y, T183Y, C240Y, C255Y, C273Y, C345Y, T366Y, T387Y, G408R, C411Y, A438R & MT542754 \\
\hline & & 1 & L40508 & 76-491 & C123Y, T135Y, C240Y, C255Y, C273Y, C291Y, C345T, T366C, C372Y, T387Y, A438G & MT542755 \\
\hline & & 1 & L40508 & 76-491 & T135Y, C159Y, T183Y, G186R, C240Y, C255Y, C273Y, C345Y, T366Y, T387Y, G408R, C423Y, A438R & MT542756 \\
\hline & & 1 & L40508 & $84-491$ & T135Y, C159Y, T183Y, G186R, C240Y, C255Y, C273Y, C345Y, T366Y, T387Y, C423Y, A438R & MT542757 \\
\hline & & 1 & $\mathrm{~L} 40508$ & $82-491$ & T135Y, C159Y, T183Y, G186R, C255Y, C273Y, C345Y, T366Y, T387C, G408R, C411Y, A438R & MT542758 \\
\hline & & 1 & L40508 & $76-491$ & T135Y, C159Y, T183Y, G186R, C255Y, C273Y, C345Y, T366Y, T387Y, C396Y, C423Y, A438R & MT542759 \\
\hline & & 1 & $\mathrm{~L} 40508$ & $84-491$ & T135Y, C177Y, T183Y, C255Y, C273Y, C345Y, T366Y, T387Y, G390R, G408R, C411Y, A438R & MT542760 \\
\hline & & 1 & L40508 & 76-491 & T135Y, T183Y, C255Y, C273Y, T366Y, T387C, G408R, A438R & MT542761 \\
\hline & & 1 & L40508 & $76-491$ & C159Y, T183Y, G186R, C255Y, C273Y, C345Y, T366Y, T387Y, A438R & MT542762 \\
\hline
\end{tabular}


Table 5. Cont.

\begin{tabular}{|c|c|c|c|c|c|c|}
\hline Assemblage & Sub-Assemblage & No. of Isolates & Reference Sequence & Stretch & Single Nucleotide Polymorphisms & GenBank ID \\
\hline & & 1 & L40508 & $76-491$ & C159Y, T183C, G186R, C255Y, C273Y, C345Y, T366Y, T387Y, C423Y, A438R & MT542763 \\
\hline & & 1 & L40508 & $76-491$ & C159Y, T183Y, G186R, C255Y, C273Y, C345Y, T366Y, T387Y, A438R, A476R & MT542764 \\
\hline & & 1 & L40508 & $76-491$ & T183C, G186A, C240Y, C255Y, C273Y, T366Y, T387C, A438R & MT542765 \\
\hline
\end{tabular}

Table 6. Diversity, frequency, and main molecular features of G. duodenalis sequences at the bg locus generated in the present study. GenBank accession numbers are provided.

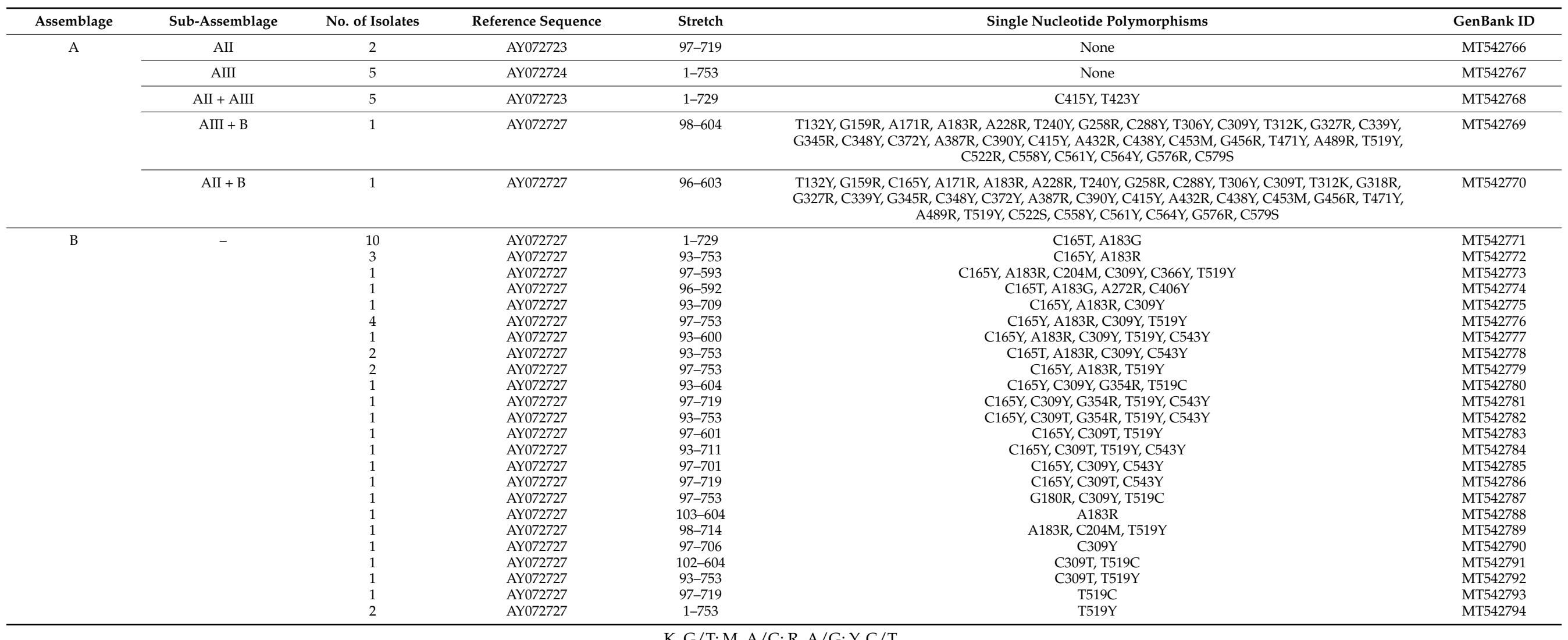

$\mathrm{K}, \mathrm{G} / \mathrm{T} ; \mathrm{M}, \mathrm{A} / \mathrm{C} ; \mathrm{R}, \mathrm{A} / \mathrm{G} ; \mathrm{Y}, \mathrm{C} / \mathrm{T}$. 
Table 7. Diversity, frequency, and main molecular features of G. duodenalis sequences at the tpi locus generated in the present study. GenBank accession numbers are provided.

\begin{tabular}{|c|c|c|c|c|c|c|}
\hline Assemblage & Sub-Assemblage & No. of Isolates & Reference Sequence & Stretch & Single Nucleotide Polymorphisms & GenBank ID \\
\hline \multirow[t]{5}{*}{ A } & AII & 8 & U57897 & 294-805 & None & MT542795 \\
\hline & & 5 & U57897 & $275-805$ & $\mathrm{C} 287 \mathrm{G}$ & MT542796 \\
\hline & & 1 & U57897 & $275-805$ & $\mathrm{C} 287 \mathrm{G}, \mathrm{A} 381 \mathrm{M}$ & MT542797 \\
\hline & $\mathrm{AII}+\mathrm{BIII}$ & 1 & U57897 & $289-754$ & 94 SNPs & MT542798 \\
\hline & & 1 & U57897 & $313-720$ & $95 \mathrm{SNPs}$ & MT542799 \\
\hline \multirow[t]{27}{*}{ B } & BIII & 7 & AF069561 & $1-456$ & None & MT542800 \\
\hline & & 1 & AF069561 & $1-456$ & A8R, C108T, C111Y, C201Y & MT542801 \\
\hline & & 1 & AF069561 & $1-456$ & A8R, C108Y, C201Y & MT542802 \\
\hline & & 2 & AF069561 & $1-456$ & A10R & MT542803 \\
\hline & & 1 & AF069561 & $1-456$ & A10R, C108Y, C111Y & MT542805 \\
\hline & & 1 & AF069561 & $1-456$ & C15Y, C33Y, C34Y, G105R, C111Y, G254R & MT542806 \\
\hline & & 1 & AF069561 & $1-456$ & $\mathrm{G} 21 \mathrm{~A}, \mathrm{C} 34 \mathrm{~T}, \mathrm{C} 108 \mathrm{~T}$ & MT542807 \\
\hline & & 1 & AF069561 & $26-456$ & C34Y, G105R, T314Y & MT542808 \\
\hline & & 1 & AF069561 & $1-456$ & G48A, G391A & MT542809 \\
\hline & & 1 & AF069561 & $17-456$ & G105R, C108Y, C201Y, G391R, & MT542810 \\
\hline & & 1 & AF069561 & $1-456$ & $\mathrm{C} 108 \mathrm{Y}$ & MT542811 \\
\hline & & 1 & AF069561 & $1-456$ & C108Y, C111Y & MT542812 \\
\hline & & 1 & AF069561 & $38-456$ & C108Y, C111Y, C201Y & MT542813 \\
\hline & & 1 & AF069561 & $1-456$ & C108Y, C201Y & MT542814 \\
\hline & BIV & 1 & AF069560 & $1-479$ & A176G, A395G, C470T & MT542817 \\
\hline & $\mathrm{BIII} / \mathrm{BIV}$ & 1 & AF069560 & $1-479$ & A5R, A33R, T57Y, C112Y, T131Y, T134Y, A176G, G281R, T314Y, A395G, C470Y & MT542818 \\
\hline & & 1 & AF069560 & $1-479$ & A5R, A33R, T57Y, T131Y, T134Y, A176G, G281R, A395G, C470Y & MT542819 \\
\hline & & 1 & AF069560 & $1-479$ & A5R, T57Y, T131Y, T134Y, A176G, A181R, A395G, C470T & MT542820 \\
\hline & & 1 & AF069560 & $1-479$ & A5R, T57Y, T131Y, T134Y, A176G, G281R, A395G, A421M, C470Y & MT542821 \\
\hline & & 1 & AF069560 & $1-479$ & A5R, A33R, T57Y, G128R, T131Y, T134Y, A176G, A395G, C470Y & MT542822 \\
\hline & & 2 & AF069560 & $1-479$ & A5R, T57Y, G128R, T131Y, T134Y, A176G, A395G, C470Y & MT542823 \\
\hline & & 1 & AF069560 & $1-479$ & A5R, T57Y, G128R, T131Y, T134Y, A176G, A395G & MT542824 \\
\hline & & 1 & AF069560 & $1-479$ & A5R, T57Y, T131Y, T134Y, A176G, C237Y, A395G, C470Y, & MT542825 \\
\hline & & 1 & AF069560 & $1-479$ & A5R, G44R, T57Y, C127Y, T131Y, T134C, A176G, A395R & MT542826 \\
\hline & & 1 & AF069560 & $1-479$ & T57Y, T131Y, T134Y, A176G, G221R, G230R, A395G, C470Y & MT542827 \\
\hline & & 1 & AF069560 & 44-479 & T57Y, T131Y, T134Y, A176G, T317Y, A395G, C470Y & MT542828 \\
\hline & & 1 & AF069560 & $1-479$ & T57Y, T131Y, T134Y, A176G, A395G, A437R, A449R, C470Y, G476R & MT542829 \\
\hline
\end{tabular}


Figure 1 shows the phylogenetic tree obtained for the $g d h$ gene by maximum parsimony and Bayesian methods. All G. duodenalis sequences clustered together (monophyletic groups) with different well-supported clades ( $100 \%$ of bootstrap and 1.0 posterior probability). Two major branches were formed and included all (A-F) G. duodenalis assemblages. The sequences of indigenous people from the Brazilian Amazon clustered in branches for assemblage A ( $97 \%$ of bootstrap and 1.0 posterior probability) and B ( $100 \%$ of bootstrap and 1.0 posterior probability). In assemblage $B$, the sequences obtained in this study clustered with sub-assemblages BIII and BIV reference strains. Similar phylogenetic trees for the $b g$ and tpi sequences generated in the present study are shown in Figures S1 and $\mathrm{S} 2$, respectively.

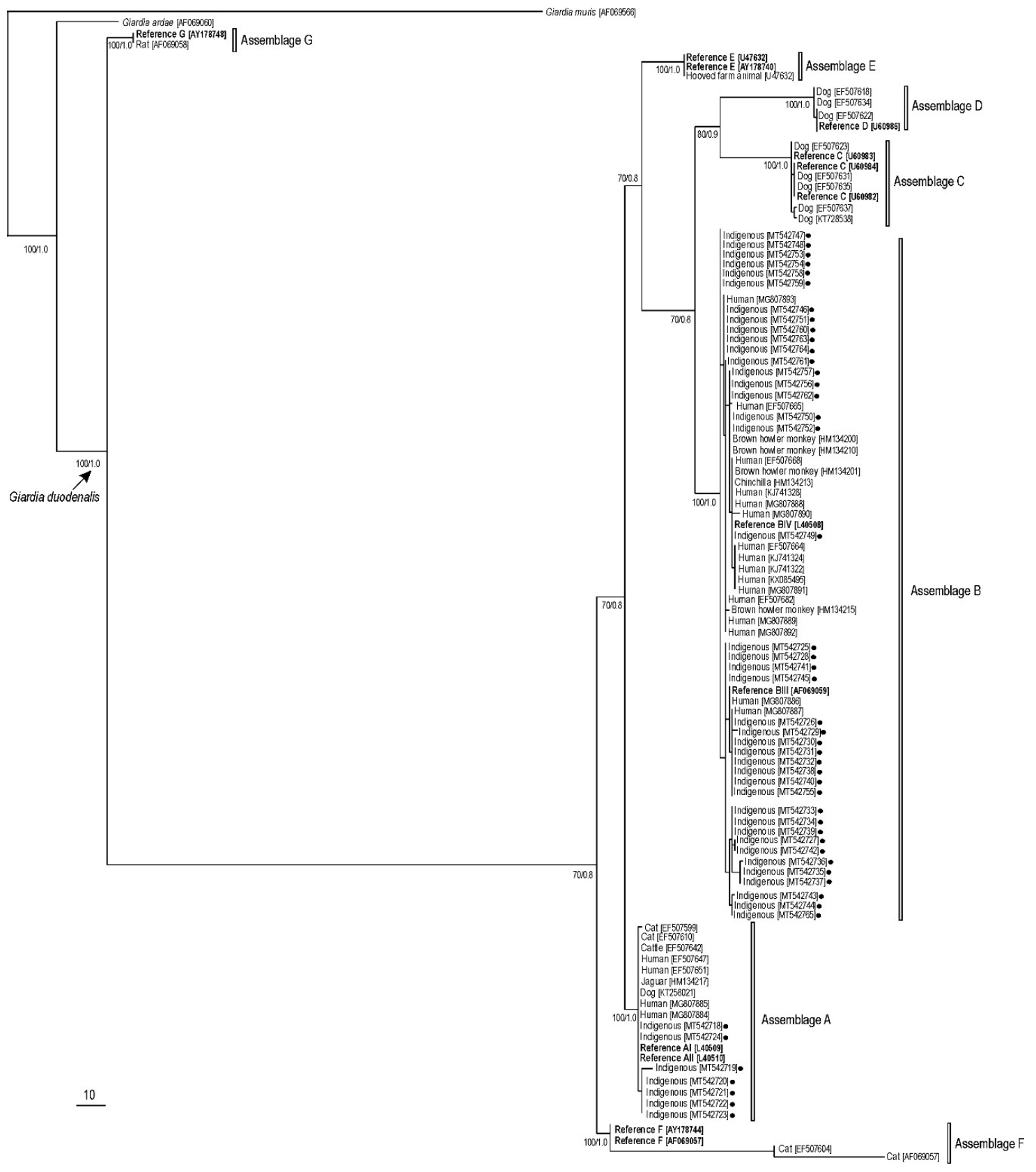

Figure 1. Maximum parsimony phylogenetic tree based on $g d h$ sequences of $G$. duodenalis. Numbers on nodes indicate the bootstrap/posterior probability values. Black filled circles represent sequences generated in the present study. GenBank accession numbers for all sequences used for the phylogenetic analysis were embedded in the tree. Giardia muris was used as the outgroup. 


\subsection{Intra-Assemblage B Genetic Diversity Analysis}

Genetic diversity was far higher within assemblage B than within assemblage A sequences regardless of the molecular marker used. Multiple sequence alignments of BIII, BIV, and ambiguous BIII/BIV sequences at the $g d h, b g$, and tpi loci revealed the presence of SNPs in multiple sites across used reference sequences, varying from 11 (for B sequences at the $b g$ locus) to 32 (for BIII/BIV sequences at the tpi locus) sites (Table S6). Overall, 611 SNPs were identified among assemblage B sequences in all three loci. Of them, $16.9 \%(103 / 611)$ corresponded to single point mutations, and $83.1 \%(508 / 611)$ to double peaks. Defined positions (hotspots) at each investigated locus tended to accumulate the bulk of these SNPs (66.9\%; 409/611). Within $g d h$, C87, T147, G150, C204, C309, and G402 were the main hotspots for BIII sequences (reference sequence: AF069059), and C123, T135, T183, G186, C255, C273, C345, T366, T387, and A438 for BIII/BIV sequences (reference sequence: L40508). Within tpi, C108 was the only hotspot for BIII sequences (reference sequence: AF069561), and A5, T57, T131, T134, A176, A395, and C470 for BIII/BIV sequences (reference sequence: AF069560). Within $b g$, the main hotspots identified for B sequences were C165, A183, C309, and T519 (reference sequence: AY072727).

The distribution of single point mutations and double peaks differed substantially among sub-assemblages and loci. At the $g d h$ locus, hotspot sites accumulated $57.7 \%$ of all SNPs detected in BIII sequences, but this figure increased to $72.9 \%$ in BIII/BIV sequences. Double peaks accounted for $37.8 \%$ of the SNPs detected in BIII sequences, but for $67.8 \%$ of the ambiguous BIII/BIV sequences (Figure 2A). At the tpi locus, hotspot sites accumulated 18.2\% of all SNPs detected in BIII sequences, but this figure increased to $54.5 \%$ in BIII/BIV sequences (Figure 2B). Finally, at the $b g$ locus, hotspot sites clustered $78.4 \%$ of the SNPs detected in assemblage B sequences, of which $58.1 \%$ corresponded to double peaks (Figure 2C).

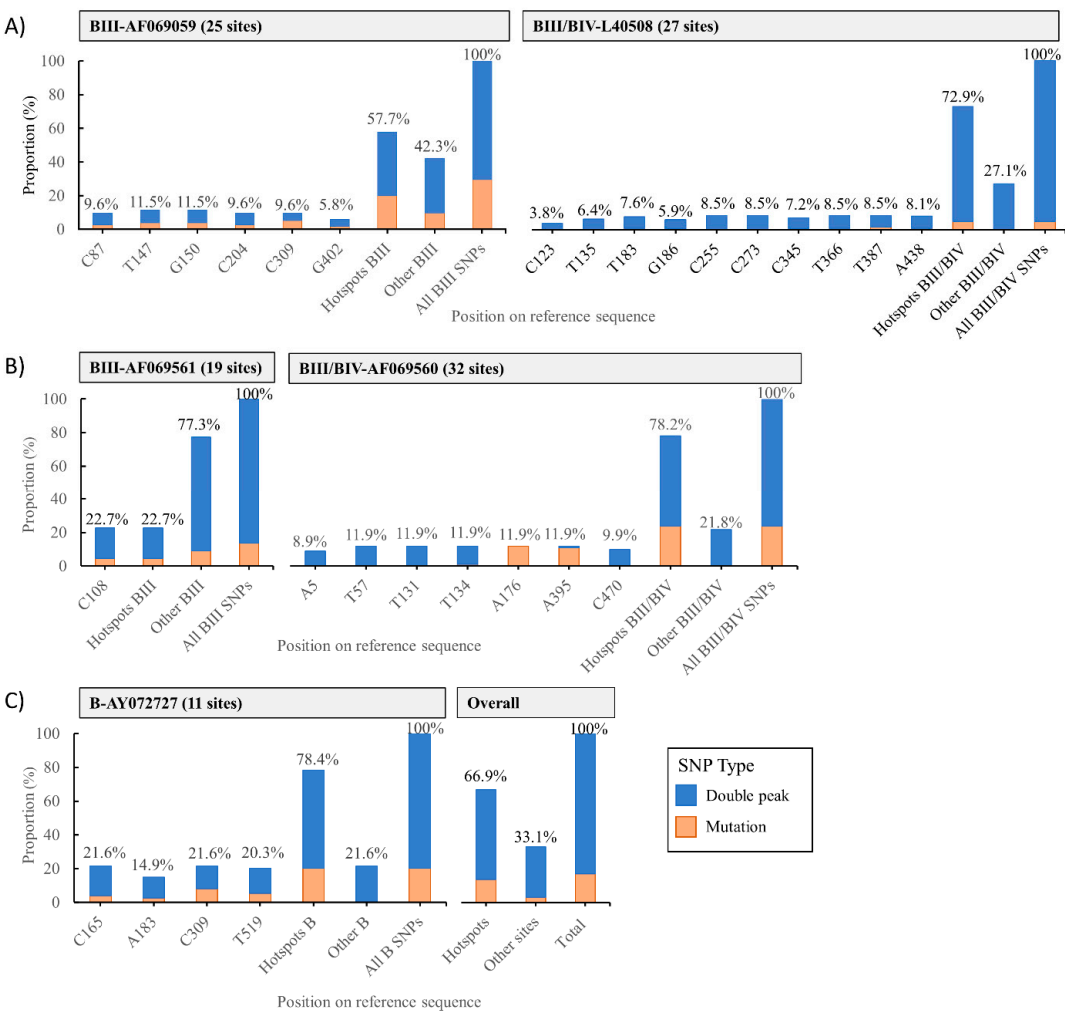

Figure 2. Distribution of single nucleotide polymorphisms segregated by mutations and double peaks, in G. duodenalis assemblage B sequences. (A) Single nucleotide polymorphisms (SNPs) at the glutamate dehydrogenase ( $g d h)$ locus; (B) SNPs at the triosephosphate isomerase (tpi); (C) SNPs at the beta-giardin (bg) locus, and overall figures for all assemblage B sequences. 


\subsection{Risk Association Analysis}

\subsubsection{Comparing G. duodenalis Negative/Ever Positive}

Overall, $55 \%$ of individuals who tested positive for G. duodenalis were female, the median age was 10 years old, with $49 \%<10$ years old. A total of $55 \%$ were from tribe 1 , followed by $15 \%$ from tribe 5 . The most frequent clinical signs were normal stool appearance $(92 \%)$, abdominal pain (53\%), and stool consistency type $2(45 \%)$. Overall, $58 \%$ did not report hand washing, $84 \%$ reported eating with hands, and $18 \%$ did not report washing fresh produce. Sanitation was predominantly defecation in the woods $(70 \%)$ and open defecation near households (21.7\%). Microscopy examination also revealed that $53 \%$ of individuals were coinfected with Endolimax nana, 48\% with Entamoeba coli, 18\% with Chilomastix mesnili, $16 \%$ with Ancylostoma spp., and $11 \%$ with Blastocystis sp.

Children under $<15$ years old reported more frequently vomiting, abdominal pain, and abnormal (mucous, bloody, mucous-bloody) faecal appearance compared to adults. However, only differences in abdominal pain appeared significant (Chi-squared test, $p=0.016$ ). There were no differences in age or symptoms between the two assemblages A and B (Chi-squared test: age group, $p=0.552$; faecal consistency, $p=0.732$; abdominal pain, $p=1$; vomit, $p=0,953)$.

In univariable analysis, hand washing, older age, tribes 2-4, coinfection with E. coli, E. nana, and Iodamoeba were negatively associated with G. duodenalis, while tribe 5, faecal consistency 4, open defecation, and the number of samples were positively associated with G. duodenalis (Table 8). Regarding the public health features and symptoms, the multivariable model retained the number of samples, age group, tribe, faecal consistency, faecal appearance, and washing fresh produce. Older age groups had a protective effect (adjusted odds ratio $(\mathrm{aOR})=0.40,95 \%$ CI: $0.20-0.81$ in $10-14$ years old, and aOR $=0.2095 \%$, CI: $0.11-0.39$ in $\geq 15$ years old, respectively), as tribes $2-3$ compared to tribe 1 (aOR $=0.46$, $95 \%$ CI: $0.24-0.85$, aOR $=0.43,95 \%$ CI: $0.21-0.85$, aOR $=0.31,95 \%$ CI: $0.14-0.63$, respectively). The number of samples was positively associated with higher odds of a G. duodenalispositive result $(\mathrm{aOR}=1.46,95 \% \mathrm{CI}: 1.18-1.81)$, as washing fresh produce $(\mathrm{aOR}=1.95,95 \%$ CI: 1.12-3.44) and faecal consistency type $4(\mathrm{aOR}=1.84,95 \%$ CI: 1.10-3.37) (Table 9). None of the other pathogens considered was found significantly associated with G. duodenalis in the multivariable model with coinfections.

Table 8. Univariable analysis. Crude association between G. duodenalis infections and variables of interest. $p$-values marked in bold indicate numbers that are significant on the $95 \%$ confidence limit (CI).

\begin{tabular}{|c|c|c|c|c|c|c|}
\hline & G. duodenalis ( $\mathrm{N}$ & s. Ever Positive) & & & ociation & \\
\hline Variable & $0, n=366^{1}$ & $1, n=198^{1}$ & $n$ & $\mathrm{OR}^{2}$ & $95 \% \mathrm{CI}^{2}$ & $p$-Value \\
\hline Sex & & & 564 & & & \\
\hline Female & $177(62 \%)$ & $109(38 \%)$ & & - & - & \\
\hline Male & $189(68 \%)$ & $89(32 \%)$ & & 0.76 & $0.54-1.08$ & 0.13 \\
\hline Age group (years) & & & 562 & & & \\
\hline $0-4$ & $38(45 \%)$ & $46(55 \%)$ & & - & - & \\
\hline $5-9$ & $67(57 \%)$ & $50(43 \%)$ & & 0.62 & $0.35-1.08$ & 0.093 \\
\hline $10-14$ & $58(62 \%)$ & $35(38 \%)$ & & 0.5 & $0.27-0.91$ & 0.023 \\
\hline$\geq 15$ & $201(75 \%)$ & $67(25 \%)$ & & 0.28 & $0.16-0.46$ & $<0.001$ \\
\hline Unknown & 2 & 0 & & & & \\
\hline Tribe & & & 564 & & & \\
\hline 1 & $155(59 \%)$ & $108(41 \%)$ & & - & - & \\
\hline 2 & $50(73 \%)$ & $19(27 \%)$ & & 0.55 & $0.30-0.96$ & 0.041 \\
\hline 3 & $55(80 \%)$ & $14(20 \%)$ & & 0.37 & $0.19-0.67$ & 0.002 \\
\hline 4 & $62(85 \%)$ & $11(15 \%)$ & & 0.25 & $0.12-0.49$ & $<0.001$ \\
\hline
\end{tabular}


Table 8. Cont.

\begin{tabular}{|c|c|c|c|c|c|c|}
\hline & G. duodenalis ( & s. Ever Positive) & & & ssociation & \\
\hline Variable & $0, n=366^{1}$ & $1, n=198^{1}$ & $n$ & $\mathrm{OR}^{2}$ & $95 \% \mathrm{CI}^{2}$ & $p$-Value \\
\hline 5 & $20(40 \%)$ & $30(60 \%)$ & & 2.15 & $1.17-4.04$ & 0.015 \\
\hline 6 & $24(60 \%)$ & $16(40 \%)$ & & 0.96 & $0.48-1.87$ & 0.9 \\
\hline Faecal consistency & & & 564 & & & \\
\hline 1 & $101(68 \%)$ & $48(32 \%)$ & & - & - & \\
\hline 2 & $200(69 \%)$ & $89(31 \%)$ & & 0.94 & $0.61-1.44$ & 0.8 \\
\hline 3 & $9(56 \%)$ & $7(44 \%)$ & & 1.64 & $0.56-4.65$ & 0.4 \\
\hline 4 & $56(51 \%)$ & $54(49 \%)$ & & 2.03 & $1.22-3.38$ & 0.006 \\
\hline Faecal appearance & & & 564 & & & \\
\hline Normal & $336(65 \%)$ & $182(35 \%)$ & & - & - & \\
\hline $\begin{array}{l}\text { Other (mucus, } \\
\text { bloody) }\end{array}$ & $30(65 \%)$ & $16(35 \%)$ & & 0.98 & $0.51-1.83$ & $>0.9$ \\
\hline Abdominal pain & & & 563 & & & \\
\hline No & $175(65 \%)$ & $93(35 \%)$ & & - & - & \\
\hline Yes & $190(64 \%)$ & $105(36 \%)$ & & 1.04 & $0.74-1.47$ & 0.8 \\
\hline Unknown & 1 & 0 & & & & \\
\hline Vomit & & & 564 & & & \\
\hline No & $353(65 \%)$ & $192(35 \%)$ & & - & - & \\
\hline Yes & $13(68 \%)$ & $6(32 \%)$ & & 0.85 & $0.29-2.18$ & 0.7 \\
\hline $\begin{array}{l}\text { Drinking water } \\
\text { source }\end{array}$ & & & 564 & & & \\
\hline River & $1(100 \%)$ & $0(0 \%)$ & & - & - & \\
\hline Well & $1(100 \%)$ & $0(0 \%)$ & & 1.00 & $0.00-36,409$ & $>0.9$ \\
\hline Piped & $364(65 \%)$ & $198(35 \%)$ & & $1,152,197$ & $0.00-\mathrm{NA}$ & $>0.9$ \\
\hline Treated water & & & 564 & & & \\
\hline No & $350(65 \%)$ & $190(35 \%)$ & & - & - & \\
\hline Yes & $16(67 \%)$ & $8(33 \%)$ & & 0.92 & $0.37-2.13$ & 0.9 \\
\hline Hand washing & & & 564 & & & \\
\hline No & $166(59 \%)$ & $115(41 \%)$ & & - & - & \\
\hline Yes & $200(71 \%)$ & $83(29 \%)$ & & 0.60 & $0.42-0.85$ & 0.004 \\
\hline $\begin{array}{l}\text { Washing fresh } \\
\text { produce }\end{array}$ & & & 563 & & & \\
\hline No & $77(68 \%)$ & $36(32 \%)$ & & - & - & \\
\hline Yes & $289(64 \%)$ & $161(36 \%)$ & & 1.19 & $0.77-1.87$ & 0.4 \\
\hline Unknown & 0 & 1 & & & & \\
\hline Eating with & & & 563 & & & \\
\hline Hand & $301(64 \%)$ & $167(36 \%)$ & & - & - & \\
\hline Flatware & $64(67 \%)$ & $31(33 \%)$ & & 0.87 & $0.54-1.38$ & 0.6 \\
\hline Unknown & 1 & 0 & & & & \\
\hline Defecation place & & & 564 & & & \\
\hline Toilets & $35(69 \%)$ & $16(31 \%)$ & & - & - & \\
\hline Woods & $294(68 \%)$ & $139(32 \%)$ & & 1.03 & $0.56-1.98$ & $>0.9$ \\
\hline Yard & $37(46 \%)$ & $43(54 \%)$ & & 2.54 & $1.23-5.41$ & 0.013 \\
\hline Contact with animals & & & 564 & & & \\
\hline No & $55(63 \%)$ & $32(37 \%)$ & & - & - & \\
\hline Yes & $311(65 \%)$ & $166(35 \%)$ & & 0.92 & $0.57-1.49$ & 0.7 \\
\hline
\end{tabular}


Table 8. Cont.

\begin{tabular}{|c|c|c|c|c|c|c|}
\hline \multirow[b]{2}{*}{ Variable } & \multicolumn{3}{|c|}{ G. duodenalis (Negative vs. Ever Positive) } & \multicolumn{2}{|c|}{ Crude Association } & \multirow[b]{2}{*}{$p$-Value } \\
\hline & $0, n=366^{1}$ & $1, n=198^{1}$ & $n$ & $\mathrm{OR}^{2}$ & $95 \% \mathrm{CI}^{2}$ & \\
\hline Rotavirus & & & 564 & & & \\
\hline No & $365(65 \%)$ & $198(35 \%)$ & & - & - & \\
\hline Yes & $1(100 \%)$ & $0(0 \%)$ & & 0.00 & & $>0.9$ \\
\hline Ancylostoma & & & 564 & & & \\
\hline No & $283(64 \%)$ & $162(36 \%)$ & & - & - & \\
\hline Yes & $83(70 \%)$ & $36(30 \%)$ & & 0.76 & $0.49-.16$ & 0.2 \\
\hline Ascaris & & & 564 & & & \\
\hline No & $364(65 \%)$ & $195(35 \%)$ & & - & - & \\
\hline Yes & $2(40 \%)$ & $3(60 \%)$ & & 2.80 & $0.46-21.4$ & 0.3 \\
\hline Blastocystis & & & 564 & & & \\
\hline No & $305(64 \%)$ & $173(36 \%)$ & & - & - & \\
\hline Yes & $61(71 \%)$ & $25(29 \%)$ & & 0.72 & $0.43-1.18$ & 0.2 \\
\hline Chilomastix & & & 564 & & & \\
\hline No & $307(66 \%)$ & $162(34 \%)$ & & - & - & \\
\hline Yes & $59(62 \%)$ & $36(38 \%)$ & & 1.16 & $0.73-1.82$ & 0.5 \\
\hline E. coli & & & 564 & & & \\
\hline No & $141(59 \%)$ & $97(41 \%)$ & & - & - & \\
\hline Yes & $225(69 \%)$ & $101(31 \%)$ & & 0.65 & $0.46-0.93$ & 0.017 \\
\hline E. histolytica & & & 564 & & & \\
\hline No & $216(63 \%)$ & $125(37 \%)$ & & - & - & \\
\hline Yes & $150(67 \%)$ & $73(33 \%)$ & & 0.84 & $0.59-1.20$ & 0.3 \\
\hline E. nana & & & 564 & & & \\
\hline No & $129(59 \%)$ & $88(41 \%)$ & & - & - & \\
\hline Yes & $237(68 \%)$ & $110(32 \%)$ & & 0.68 & $0.48-0.97$ & 0.032 \\
\hline Hymenolepis & & & 564 & & & \\
\hline No & 333 (65\%) & $180(35 \%)$ & & - & - & \\
\hline Yes & $33(65 \%)$ & $18(35 \%)$ & & 1.01 & $0.54-1.82$ & $>0.9$ \\
\hline Iodamoeba & & & 564 & & & \\
\hline No & $336(64 \%)$ & $191(36 \%)$ & & - & - & \\
\hline Yes & $30(81 \%)$ & $7(19 \%)$ & & 0.41 & $0.16-0.90$ & 0.038 \\
\hline Isospora & & & 564 & & & \\
\hline No & $366(65 \%)$ & $197(35 \%)$ & & - & - & \\
\hline Yes & $0(0 \%)$ & $1(100 \%)$ & & $1,447,714$ & 0.00, NA & $>0.9$ \\
\hline Sarcocystis & & & 564 & & & \\
\hline No & $359(65 \%)$ & $196(35 \%)$ & & - & - & \\
\hline Yes & $7(78 \%)$ & $2(22 \%)$ & & 0.52 & $0.08-2.19$ & 0.4 \\
\hline Strongyloides & & & 564 & & & \\
\hline No & 345 (64\%) & $191(36 \%)$ & & - & - & \\
\hline Yes & $21(75 \%)$ & $7(25 \%)$ & & 0.60 & $0.23-1.38$ & 0.3 \\
\hline Taenia & & & 564 & & & \\
\hline No & $364(65 \%)$ & $198(35 \%)$ & & - & - & \\
\hline Yes & $2(100 \%)$ & $0(0 \%)$ & & 0.00 & & $>0.9$ \\
\hline
\end{tabular}


Table 8. Cont.

\begin{tabular}{|c|c|c|c|c|c|c|}
\hline \multirow[b]{2}{*}{ Variable } & \multicolumn{3}{|c|}{ G. duodenalis (Negative vs. Ever Positive) } & \multicolumn{2}{|c|}{ Crude Association } & \multirow[b]{2}{*}{$p$-Value } \\
\hline & $0, n=366^{1}$ & $1, n=198^{1}$ & $n$ & $\mathrm{OR}^{2}$ & $95 \% \mathrm{CI}^{2}$ & \\
\hline Trichuris & & & 564 & & & \\
\hline No & $365(65 \%)$ & $197(35 \%)$ & & - & - & \\
\hline Yes & $1(50 \%)$ & $1(50 \%)$ & & 1.85 & $0.07-47.0$ & 0.7 \\
\hline Cyclospora & & & 564 & & & \\
\hline No & $351(65 \%)$ & $188(35 \%)$ & & - & - & \\
\hline Yes & $15(60 \%)$ & $10(40 \%)$ & & 1.24 & $0.53-2.80$ & 0.6 \\
\hline No. of samples & & & 564 & 1.72 & $1.43-2.08$ & $<0.001$ \\
\hline 1 & $71(83 \%)$ & $15(17 \%)$ & & & & \\
\hline 2 & $99(73 \%)$ & $37(27 \%)$ & & & & \\
\hline 3 & $129(64 \%)$ & $72(36 \%)$ & & & & \\
\hline 4 & $67(48 \%)$ & $74(53 \%)$ & & & & \\
\hline
\end{tabular}

${ }^{1}$ Statistics presented: $n(\%) .{ }^{2}$ OR, crude odds ratio; CI, confidence interval; NA, not applicable.

Table 9. Multivariable analysis comparing G. duodenalis-negative results versus G. duodenalis ever positive results. $p$-values marked in bold indicate numbers that are significant on the $95 \%$ confidence limit (CI).

\begin{tabular}{|c|c|c|c|}
\hline Variable & $\mathrm{aOR}^{1}$ & $95 \% \mathrm{CI}^{1}$ & $p$-Value \\
\hline \multicolumn{4}{|l|}{ Age group (years) } \\
\hline $0-4$ & - & - & \\
\hline $5-9$ & 0.58 & $0.30-1.12$ & 0.11 \\
\hline $10-14$ & 0.40 & $0.20-0.81$ & 0.011 \\
\hline$\geq 15$ & 0.20 & $0.11-0.39$ & $<0.001$ \\
\hline Number_samples & 1.46 & $1.18-1.81$ & $<0.001$ \\
\hline \multicolumn{4}{|l|}{ Tribe } \\
\hline 1 & - & - & \\
\hline 2 & 0.46 & $0.24-0.85$ & 0.016 \\
\hline 3 & 0.43 & $0.21-0.85$ & 0.018 \\
\hline 4 & 0.31 & $0.14-0.63$ & 0.002 \\
\hline 5 & 1.83 & $0.94-3.60$ & 0.075 \\
\hline 6 & 0.94 & $0.45-1.95$ & 0.9 \\
\hline \multicolumn{4}{|l|}{ Washing fresh produce } \\
\hline No & - & - & \\
\hline Yes & 1.95 & $1.12-3.44$ & 0.020 \\
\hline \multicolumn{4}{|l|}{ Faecal consistency } \\
\hline 1 & - & - & \\
\hline 2 & 0.93 & $0.59-1.49$ & 0.8 \\
\hline 3 & 2.37 & $0.73-7.51$ & 0.14 \\
\hline 4 & 1.84 & $1.01-3.37$ & 0.046 \\
\hline \multicolumn{4}{|l|}{ Faecal appearance } \\
\hline Normal & - & - & \\
\hline Other (mucus, bloody) & 0.51 & $0.23-1.08$ & 0.087 \\
\hline
\end{tabular}

${ }_{1}^{1}$ aOR, adjusted odds ratio; $\mathrm{CI}$, confidence interval. $n=559$ (removing five observations with missing values). 


\subsubsection{Comparing G. duodenalis Serial Results}

Out of the 478 observations with more than one sample, 62\% (295/478) always tested negative for G. duodenalis, $4 \%(20 / 478)$ always tested positive, and 34\% (163/478) were discontinuously positive. As such, among observations ever positive for G. duodenalis, 11\% $(20 / 183)$ were continuously positive. Of those, $70 \%$ were aged $0-9$ years old, $55 \%$ were from tribe 1, and 35\% from tribe 5 . Overall, $85 \%$ did not report hand washing, $30 \%$ not washing fresh produce, $50 \%$ reported open defecation near the households, and $45 \%$ open defecation in the woods. However, in the multivariable analysis, discontinuous positivity was strongly associated with the number of samples (aOR $=0.30,95 \% \mathrm{CI}: 0.12-0.66)$, and tribe $5(\mathrm{aOR}=4.76,95 \% \mathrm{CI}: 1.30-18.6)$, but no further significant association was found (Tables S7 and S8).

Finally, comparing observations that were always negative versus always positive, the multivariable model only retained age group and tribe, showing evidence of a protective effect of older age groups ( $\mathrm{aOR}=0.24,95 \% \mathrm{CI}$ : $0.06-0.89$ for children 5-9 years old; aOR $=0.17,95 \%$ CI: $0.03-0.68$ for children $10-14$ years old; $\mathrm{aOR}=0.05,95 \%$ CI: $0.01-0.20$ for children $\geq 15$ years old), and a strong positive association with tribe 5 ( $a \mathrm{OR}=5.55,95 \%$ CI: 1.61-19.4) (Tables S9 and S10). However, when adjusting for the effect of coinfections; the best fit model suggested a protective effect of E. nana ( $\mathrm{aOR}=0.25,95 \%$ CI: 0.08-73), oldest age group $\geq 15$ years old ( $\mathrm{aOR}=0.07,95 \%$ CI: $0.01-0.28$ ), and a positive effect of tribe $5(\mathrm{aOR}=5.87,95 \%$ CI: $1.60-22.1)($ Table S11).

\section{Discussion}

This survey presents new insights into the epidemiology of G. duodenalis in Amazonian indigenous communities. The main contributions of the study include the demonstration that (i) giardiasis is a common finding (13-22\%) in apparently healthy Tapirapé people, mainly affecting children in the age group of 0-9 years old; (ii) assemblage B was responsible for near $70 \%$ of the mostly asymptomatic infections detected; and (iii) a high degree of genetic heterogeneity was observed within assemblage $B$ (but not assemblage A) sequences, regardless of the molecular marker used.

Several epidemiological studies conducted in endemic areas worldwide have shown that G. duodenalis infections do not seem to correlate positively with diarrhoea [23,24], demonstrating that asymptomatic giardiasis is the rule rather than the exception in these settings. This fact would explain why giardiasis is systematically absent in global burden estimations of diarrhoeal disease [25]. This seems to be also the case of the present study, where G. duodenalis infections were detected similarly in asymptomatic individuals (33.8\%) and individuals presenting with diarrhoea or other gastrointestinal manifestations (35.3\%). Taken together, this information supports the hypothesis that some enteric protist species (e.g., Blastocystis sp., Dientamoeba fragilis, G. duodenalis) might in fact be protective against disease [26]. This is an attractive possibility implying that these agents are indeed acting as pathobionts (that is, microorganisms that normally live as harmless symbionts but under certain circumstances can be pathogenic) forming part of the host eukaryome.

We have shown in our study that $G$. duodenalis infection was strongly related to younger age and tribe (with tribes 1 and 5 having a higher association) and to seasonality. This may be due to external factors associated with indirect transmission pathways of the infection (e.g., source of drinking water, consumption of contaminated fresh produce, swimming in contaminated surface waters, defecation on the open ground near households, and high density of companion or domestic animals) or increased risk of reinfection within the tribe from other infected members through direct person-to-person contact. Contact with faecally contaminated water and produce may be more likely in the rainy season. Children $<15$ years old with giardiasis reported more frequently vomiting, abdominal pain, and presence of mucus/blood in faeces compared to adults, although observed differences did not reach statistical significance. Young children with an immature immune system may be at higher risk of infections and probably more severe disease episodes. Thus, older adults may have acquired immunity after a previous infection. Indeed, it has been 
shown that levels of intestinal inflammation caused by G. duodenalis infection decrease with subsequent infections [27,28]. This implies that there is acquired protection against the severity of giardiasis but not from reinfection [29]. In this regard, it should be noted that the composition and abundance of the host's microbiota have also been suggested to play an important role in the outcome of the infection [30].

Giardiasis was also strongly dependent on the number of samples taken, even considering that conventional microscopy (a method that is largely known to be of limited diagnostic sensitivity) was the screening method for the initial detection of G. duodenalis in the present survey. This suggests that possible reinfections or chronic infections with intermittent positivity may be more common than initially anticipated. Reinfection may be more pronounced in the rainy season. In addition, no evident differences between individuals continuously positive/discontinuously positive to G. duodenalis were found. However, we should exclude a bias in those presenting for sampling. This is unlikely to be a major factor due to the lack of symptoms in most cases.

Regarding coinfections, the presence of $G$. duodenalis was not associated with any other enteric parasite species, except possibly E. nana. These results may be biased by the relatively small number of positive samples detected for certain pathogens and should, therefore, be interpreted with caution. Similarly, a counter-intuitive positive association between $G$. duodenalis with washing fresh produce was found. This result may be the consequence of the potential confounder effect of other variables no considered here such as the manipulation of fresh produce or the use of contaminated washing water. The latter possibility would support the relevance of waterborne transmission for human giardiasis.

Molecular sequence analyses of the three loci used here for genotyping purposes also revealed interesting data. There were no differences in age between individuals infected either by the assemblage A or the assemblage B of G. duodenalis. Regarding age-related patterns in the distribution of G. duodenalis assemblages, our results are in contrast with those previously obtained in surveys targeting clinical populations. For instance, children have been shown to be more commonly infected by assemblage B ( $83 \%$, $44 / 53)$ than adults $(52 \%, 22 / 42)$ in patients of all age groups in Spain [31]. Moreover, in that country, assemblage B was significantly more prevalent than assemblage A in asymptomatic outpatient children, but not in individuals of older age [32].

Remarkably, no association between the occurrence of diarrhoea (or any other gastrointestinal manifestation) and the G. duodenalis assemblage involved in the infection was found in the investigated population. This result corroborates that observed in children under 5 years of age $(n=222)$ recruited under the Global Enteric Multicentre Study (GEMS) in Mozambique [33]. However, it should be noted that other surveys have shown different, even contradictory, results. For instance, assemblage A was more prevalent than assemblage B in Bangladeshi people $(n=343)$ [34], in Turkish clinical patients $(n=44)$ [35], and in Spanish outpatient children $(n=43)$ [32]. The opposite trend was reported in asymptomatic infected individuals $(n=18)$ in the Netherlands [36].

Genotyping data generated here demonstrated that assemblage B was responsible for three out of four G. duodenalis infections in the Tapirapé people, a similar proportion of that $(78 \%)$ described in paediatric populations in the Amazonas State [37]. Of note, assemblage A tends to be the predominant $G$. duodenalis genetic variant circulating in humans in Brazil (Table S1). These facts may be indicative of differences in sources of infection, transmission pathways, or even geographical segregation patterns of the parasite in the country. Lack of non-human, host-specific assemblages C-F seem to suggest that companion, production, and free-living animal species are no significant contributors of giardiasis in the surveyed population. This is in spite of the fact that swine and poultry were reared in all seven tribes, and that domestic dog and cat densities were also high. In addition, cattle (but not sheep) farming was also frequent in the proximity of them. Taking together, these data indicate that human giardiasis is mainly of anthropic nature among the Tapirapé people. The extent and accuracy of this statement should be corroborated in future molecular epidemiological studies including animal and environmental (water) samples. 
This study also confirms the high genetic variability within G. duodenalis assemblage B (but not assemblage A) reported frequently in similar molecular epidemiological surveys conducted in endemic areas globally [38,39] including Brazil $[40,41]$. This finding was particularly evident at the $g d h$ and tpi loci, for which most of the generated BIII (78-87\%), BIV (100\%), and BIII/BIV (90-92\%) sequences corresponded to distinct genotypes of the parasite. Sequences unmistakably assigned to BIII and BIV at the $g d h / t p i$ loci tended to vary only in one to six positions (hotspots) either as mutations or ambiguous (double peak) sites. In these sets of hotspots, the proportion of sites involving double peaks in BIII sequences varied from $38 \%$ at the $g d h$ locus to $18 \%$ at the tpi locus. Interestingly, these percentages increased in both cases to 55-68\% in ambiguous BIII/BIV sequences, explaining why these isolates were difficult to allocate to a given sub-assemblage. Two independent mechanisms have been proposed to explain the presence of ambiguous (double peak) positions. The first one involves the occurrence of true mixed infections (e.g., BIII + BIV) and would fit well with an epidemiological scenario characterised by high infection and reinfection rates as the one described in the present study. The second one would be associated with the occurrence of genetic recombination. Evidence for the latter possibility comes from independent investigations demonstrating low levels of allelic sequence heterozygosity (implying a genetic homogenisation mechanism) within assemblage A [42] and, to a lesser extent, within assemblage B [43]. Additional evidence of genetic recombination events has been demonstrated within assemblage B in single (trophozoite and cyst) cells [44] and within sub-assemblages BIII and BIV at the genetic population level [45].

The results obtained in the present study may be biased by certain design and methodological constricts. For instance, the initial screening of G. duodenalis was based on conventional microscopy, so the true prevalence of the infection is likely to be underestimated. In addition, there may be a response bias as people may be more or less inclined to return to the study if they had a negative or positive test result. Interestingly, the positivity rate was increased by the number of tests performed, suggesting that over time people were likely to have had a giardiasis episode, that they may have had a false-negative result at microscopy examination, or an inherent response bias in that people who were likely to be positive would return for testing. Limitations associated with the main dataset may arise from the combination of period-specific data, although most of the independent variables considered (e.g., demographics, access to safe drinking water, and sanitary conditions) were not expected to change over time. As our analyses used the first negative test result, we could not further explore the effect of seasonality in the multivariable analysis. However, we have already shown in the descriptive data that seasonality is associated with infections and repeated infections. Lack of association between $G$. duodenalis genetic variants and occurrence of clinical symptoms may be influenced by the fact that other diarrhoea-causing agents (including viral and bacterial pathogens) were not assessed. In addition, suspected mixed infections were not further investigated by cloning of PCR amplicons or next-generation sequencing, methods with high sensitivity able to detect genetic variants of the parasite that are underrepresented in the population pool, and that are otherwise undetectable using conventional PCR methods and Sanger sequencing. Finally, the typing scheme used in the present study may lack enough phylogenetic resolution to correctly differentiate between sub-assemblage BIII and BIV sequences. This issue has been highlighted in recent molecular studies for assemblage B and assemblage A sequences [46,47]. This important point emphasises the need of identifying new markers and of developing novel methods for MLST purposes.

\section{Materials and Methods}

\subsection{Study Area}

Brazil extends over $8,511,965 \mathrm{~km}^{2}$ and includes 724 indigenous lands (ILs) covering a total area of $1,173,770 \mathrm{~km}^{2}$ and accounting for $14 \%$ of the country's territory [48]. Most ILs are concentrated in the Legal Amazon, representing 23\% of the Amazon territory) [49]. The indigenous people from the Tapirapé ethnicity live in the Serra do Urubu Branco region, 
Mato Grosso State, a region of tropical forest with typical Amazonian flora and fauna interspersed with clean and closed fields. The Tapirapé exploit this environment alternating agriculture, hunting, gathering, and fishing according to the time of year $[49,50]$. Farmers 'villages have traditionally been in the vicinity of dense forests on high, non-flooding lands. Currently, the Tapirapé ethnic group is made up of approximately 700 individuals living in six tribes with maximum and minimum distances from the main tribe of $70 \mathrm{~km}$ and $10 \mathrm{~km}$, respectively. The main tribe is in the municipality of Confresa, Mato Grosso State (Figure 3). Tapirapé people interact frequently with individuals from other ethnic tribes at social events, hunt parties, and other activities.



Figure 3. Map showing the exact geographical location of the sites sampled in the present study.

\subsection{Sampling and Data Collection}

This is a prospective, cross-sectional epidemiological study including four sampling periods covering two dry (July 2008 and July 2009) and two rainy (January 2009 and January 2010) seasons. After obtaining the chief's ('cacique') consent for permission to survey, all members of the tribe were informed about the aim of the project and invited to provide a single stool sample at each of the four scheduled sampling periods. Designated persons in each household were given polystyrene plastic flasks for each member of the household and stool samples were collected on the following day.

Individual standardised questionnaires were completed by a member of our research team in face-to-face interviews with designated persons at sample collection, who provided the requested information for each member of his/her household. Questions included demographics (gender, age, village of origin), clinical manifestations (vomit, abdominal pain), hand and vegetable washing, source of drinking water, use of water treatment, defecation place, and contact with domestic animals and livestock. Provided stool samples were visually inspected for consistency and the presence of mucus or blood. Each participant 
was assigned a unique distinctive code through the whole period of study, which was used to identify his/her stool sample(s) and associated epidemiological questionnaire(s).

\subsection{Microscopy Examination}

Stool samples were kept at $4{ }^{\circ} \mathrm{C}$ before microscopy examination, usually within $48 \mathrm{~h}$ of collection. A conventional flotation method using sucrose solution (specific gravity: $1.2 \mathrm{~g} / \mathrm{cm}^{3}$ ) was conducted in all stool samples as previously described [51]. Two additional techniques were performed-spontaneous sedimentation [52] and centrifugalsedimentation in formalin-ether [53]. A sample was considered G. duodenalis-positive if cysts of the parasite were detected by at least one of the three methods used. Aliquots of faecal positive samples were stored at $-20^{\circ} \mathrm{C}$ for downstream molecular analyses. Any other enteric parasite (including helminthic and protist) species found during microscopy observation were also identified and recorded.

\subsection{DNA Extraction and Purification}

Positive stool samples were defrosted and G. duodenalis cysts concentrated and purified using the Faust method [54]. Obtained supernatants were subjected to three freeze-thaw cycles to facilitate the mechanical breakage of the cyst wall [55]. Genomic DNA was extracted from the processed supernatants (ca $200 \mu \mathrm{L}$ ) using the PureLink Genomic DNA Mini Kit (Thermo Fisher Scientific, Waltham, MA, USA) according to the manufacturer's instructions. Extracted and purified DNA samples in molecular grade water $(200 \mu \mathrm{L})$ were kept at $-20{ }^{\circ} \mathrm{C}$ and shipped to the Spanish National Centre for Microbiology (Health Institute Carlos III) in Majadahonda (Spain) for downstream genotyping analyses.

\subsection{Molecular Confirmation of G. duodenalis}

Confirmation of $G$. duodenalis infection was achieved using a real-time PCR (qPCR) method targeting a 62-bp region of the gene codifying the small subunit ribosomal RNA (SSU rRNA) of the parasite [56]. Amplification reactions $(25 \mu \mathrm{L})$ consisted of $3 \mu \mathrm{L}$ of template DNA, $0.5 \mu \mathrm{M}$ of each primer Gd-80F and Gd-127R, $0.4 \mu \mathrm{M}$ of the probe (Table S12), and 12.5 $\mu \mathrm{L}$ TaqMan ${ }^{\circledR}$ Gene Expression Master Mix (Applied Biosystems, Foster City, CA, USA). Detection of parasitic DNA was performed on a Corbett Rotor GeneTM 6000 realtime PCR system (Qiagen, Hilden, Germany) using an amplification protocol consisting of an initial hold step of $2 \mathrm{~min}$ at $55^{\circ} \mathrm{C}$ and $15 \mathrm{~min}$ at $95^{\circ} \mathrm{C}$, followed by 45 cycles of $15 \mathrm{~s}$ at $95^{\circ} \mathrm{C}$ and $1 \mathrm{~min}$ at $60^{\circ} \mathrm{C}$. Water (no template) and genomic DNA (positive) controls were included in each PCR run.

\subsection{Molecular Characterisation of G. duodenalis}

Giardia duodenalis isolates with a qPCR-positive result were re-assessed by sequencebased multi-locus genotyping of the genes encoding for the glutamate dehydrogenase (gdh), beta-giardin (bg), and triosephosphate isomerase (tpi) proteins of the parasite. A semi-nested PCR was used to amplify a $\sim 432$-bp fragment of the gdh gene [57]. PCR reaction mixtures $(25 \mu \mathrm{L})$ included $5 \mu \mathrm{L}$ of template DNA and $0.5 \mu \mathrm{M}$ of the primer pairs $\mathrm{GDHeF} / \mathrm{GDHiR}$ in the primary reaction and GDHiF/GDHiR in the secondary reaction (Table S12). Both amplification protocols consisted of an initial denaturation step at $95{ }^{\circ} \mathrm{C}$ for $3 \mathrm{~min}$, followed by 35 cycles of $95^{\circ} \mathrm{C}$ for $30 \mathrm{~s}, 55^{\circ} \mathrm{C}$ for $30 \mathrm{~s}$, and $72{ }^{\circ} \mathrm{C}$ for $1 \mathrm{~min}$, with a final extension of $72{ }^{\circ} \mathrm{C}$ for $7 \mathrm{~min}$.

A nested PCR was used to amplify a $\sim 511$ bp-fragment of the $b g$ gene [58]. PCR reaction mixtures $(25 \mu \mathrm{L})$ consisted of $3 \mu \mathrm{L}$ of template DNA and $0.4 \mu \mathrm{M}$ of the primers sets G7_F/G759_R in the primary reaction and G99_F/G609_R in the secondary reaction (Table S12). The primary PCR reaction was carried out with the following amplification conditions: one step of $95^{\circ} \mathrm{C}$ for $7 \mathrm{~min}$, followed by 35 cycles of $95^{\circ} \mathrm{C}$ for $30 \mathrm{~s}, 65^{\circ} \mathrm{C}$ for $30 \mathrm{~s}$, and $72{ }^{\circ} \mathrm{C}$ for $1 \mathrm{~min}$, with a final extension of $72{ }^{\circ} \mathrm{C}$ for $7 \mathrm{~min}$. The conditions for the secondary PCR were identical to the primary PCR except that the annealing temperature was $55^{\circ} \mathrm{C}$. 
A nested PCR was used to amplify a $~ 530 \mathrm{bp}$-fragment of the tpi gene [59]. PCR reaction mixtures $(50 \mu \mathrm{L})$ included $2-2.5 \mu \mathrm{L}$ of template DNA and $0.2 \mu \mathrm{M}$ of the primer pairs AL3543/AL3546 in the primary reaction and AL3544/AL3545 in the secondary reaction (Table S12). Both amplification protocols consisted of an initial denaturation step at $94{ }^{\circ} \mathrm{C}$ for $5 \mathrm{~min}$, followed by 35 cycles of $94{ }^{\circ} \mathrm{C}$ for $45 \mathrm{~s}, 50{ }^{\circ} \mathrm{C}$ for $45 \mathrm{~s}$, and $72{ }^{\circ} \mathrm{C}$ for $1 \mathrm{~min}$, with a final extension of $72{ }^{\circ} \mathrm{C}$ for $10 \mathrm{~min}$.

The semi-nested and nested PCR protocols described above were conducted on a 2720 Thermal Cycler (Applied Biosystems). Reaction mixes always included 2.5 units of MyTAQ $^{\mathrm{TM}}$ DNA polymerase (Bioline $\mathrm{GmbH}$, Luckenwalde, Germany), and 5× MyTAQTM reaction buffer containing $5 \mathrm{mM}$ dNTPs and $15 \mathrm{mM} \mathrm{MgCl}_{2}$. Laboratory-confirmed positive and negative DNA samples for each parasite species investigated were routinely used as controls and included in each round of PCR. PCR amplicons were visualised on $2 \%$ D5 agarose gels (Conda, Madrid, Spain) stained with Pronasafe nucleic acid staining solution (Conda). Positive PCR products were directly sequenced in both directions using appropriate internal primer sets (Table S12). DNA sequencing was conducted by capillary electrophoresis using BigDye ${ }^{\circledR}$ Terminator chemistry (Applied Biosystems) on an on ABI PRISM 3130 Genetic Analyser.

\subsection{Sequence and Phylogenetic Analyses}

Raw sequencing data in both forward and reverse directions were viewed using the Chromas Lite version 2.1 sequence analysis program (https://technelysium.com. au/wp/chromas/ (accessed on 1 February 2021)). The Basic Local Alignment Search Tool (BLAST) (http://blast.ncbi.nlm.nih.gov/Blast.cgi (accessed on 1 February 2021)) was used to compare nucleotide sequences with sequences retrieved from the NCBI GenBank database. Generated DNA consensus sequences were aligned to appropriate reference sequences using the MEGA 6free software [60] for species confirmation and assemblage/sub-assemblage identification.

For the estimation of the phylogenetic relationships among the identified Giardiapositive samples, gdh sequences generated in this study and human- and animal-derived homologue sequences mostly from Brazil retrieved from GenBank were aligned using Clustal $X$ and adjusted manually with GeneDoc [61,62]. Inferences by maximum parsimony (MP) were constructed by PAUP version 4.0b10 using a heuristic search in 1000 replicates, 500 bootstrap replicates, random stepwise addition starting trees (with random addition sequences), and tree bisection and reconnection branch swapping [63]. MrBayes v3.1.2 was used to perform Bayesian analyses with four independent Markov chain runs for 1,000,000 metropolis-coupled MCMC generations, sampling a tree every 100th generation [64]. References [65-109] are cited in the supplementary materials. The first $25 \%$ of trees represented burn-in and the remaining trees were used to calculate Bayesian posterior probability. The GTR $+\mathrm{I}+\mathrm{G}$ substitution model was used. The gdh sequence of $\mathrm{G}$. ardeae was used as the outgroup.

The sequences obtained in this study have been deposited in GenBank under accession numbers MT542718-MT542765 (gdh), MT542766-MT542794 (bg), and MT542795-MT542829 (tpi).

\subsection{Statistical Analysis}

We investigated factors (public health features, clinical symptoms, coinfection with other pathogens) associated with a positive G. duodenalis result. The main dataset was constructed with data from one of the four sampling points-if the observation ever tested positive for $G$. duodenalis, we used data from the sampling point of the first positive $G$. duodenalis result; otherwise, we used data from the first sampling point in order.

We conducted Chi-squared tests $(p<0.05)$ to compare characteristics of cases and non-cases, and we calculated crude odds ratios (OR) with $95 \%$ confidence intervals (CI) to investigate the crude association between independent variables and a G. duodenalispositive result. We constructed multivariable logistic regression models to assess the association between G. duodenalis and (i) public health features and clinical signs or (ii) 
coinfection with other intestinal pathogens, adjusted by age, tribe, and the number of samples. Additionally, we considered the serial results of G. duodenalis for observations with at least two samples. We conducted similar analyses by comparing those continuously negative versus continuously positive, and those discontinuously positive versus continuously positive.

Univariable analyses were conducted on all available observations, but observations with missing values were removed from multivariable analyses. All the independent variables were included in the analyses and we used the stepwise backward selection method, removing successively the least significant variable and using Akaike information criterion (AIC) and Bayesian information criterion (BIC) to construct the best fit model. Analyses were performed in R (package stats).

\subsection{Ethics Approval}

This study has been approved by the National Research Ethics Commission (CONEP), Ministry of Health (Brazil), under reference number 120/2008.

\section{Conclusions}

This microscopy-based survey demonstrates that symptomatic and asymptomatic giardiasis are common in indigenous people from the Brazilian Amazon. Children under 15 years of age were particularly exposed to the infection, suggesting that acquired immunity plays a role in modulating the frequency and virulence of the disease. G. duodenalis infection rates varied largely among the surveyed tribes and sampling periods, suggesting that different pathways may be involved in the transmission of the parasite. Molecular sequence data indicated that the most likely source of infection was anthropic. The distribution of assemblages was independent of the occurrence of clinical manifestations, indicating that the genotype of the parasite was not associated with the outcome of the infection. Assemblage B accounted for near $75 \%$ of the infections detected and showed a high genetic diversity that impaired the correct identification of sub-assemblages BIII and BIV. This diversity was mainly associated with the presence of ambiguous positions (double peaks) at the chromatogram level, suggesting that coinfections and/or genetic recombination events were taking place, at unknown rates, in the investigated population. Further molecular epidemiological studies targeting animal (including domestic and wildlife) and environmental (drinking water) samples are needed to elucidate the transmission dynamics of $G$. duodenalis in this Brazilian geographical region.

Supplementary Materials: The following are available online at https:/ / www.mdpi.com/2076-0 817/10/2/206/s1, Table S1: Prevalence and molecular diversity of Giardia duodenalis in humans in Brazil, Table S2: Prevalence and molecular diversity of Giardia duodenalis in domestic and wildlife animal species in Brazil, Table S3: Prevalence and molecular diversity of Giardia duodenalis in water samples in Brazil, Table S4: Prevalence and molecular diversity of Giardia duodenalis in fresh produce in Brazil, Table S5: Full dataset showing the molecular diversity of G. duodenalis at the $g d h, b g$, and tpi molecular markers, Table S6: Intra-assemblage B single nucleotide polymorphisms distribution and classification among $G$. duodenalis sequences at the $g d h, b g$ and $t p i$ loci. Hotspots for SNPs are identified and the summarised comparisons of frequencies between the hotspot and non-hotspot sites are highlighted with darker shades, Table S7: Univariable analysis comparing discontinuously G. duodenalis-positive results versus continuously G. duodenalis-positive results. $p$-values marked in bold indicate numbers that are significant on the 95\% confidence limit, Table S8: Multivariable analysis comparing discontinuously G. duodenalis-positive results versus always $G$. duodenalis-positive results. $p$-values marked in bold indicate numbers that are significant on the $95 \%$ confidence limit, Table S9: Univariable analysis comparing always $G$. duodenalis-negative results versus always $G$. duodenalis-positive results. $p$-values marked in bold indicate numbers that are significant on the 95\% confidence limit, Table S10: Multivariable analysis always comparing G. duodenalis-negative results versus always $G$. duodenalis-positive results. $p$-values marked in bold indicate numbers that are significant on the $95 \%$ confidence limit, Table S11: Multivariable analysis comparing always G. duodenalis-negative results versus always $G$. duodenalis-positive results and considering the presence 
of coinfections. $p$-values marked in bold indicate numbers that are significant on the $95 \%$ confidence limit, Table S12: Oligonucleotides used for the molecular identification and characterisation of $G$. duodenalis in the present study, Figure S1: Maximum parsimony phylogenetic dendogram based on bg sequences of $G$. duodenalis. Numbers on nodes indicate the bootstrap/posterior probability values. GenBank accession numbers for all sequences used for the phylogenetic analysis were embedded in the tree, Figure S2: Maximum parsimony phylogenetic dendogram based on tpi sequences of G. duodenalis. Numbers on nodes indicate the bootstrap/posterior probability values. GenBank accession numbers for all sequences used for the phylogenetic analysis were embedded in the tree.

Author Contributions: Conceptualisation, A.F.M., J.J.S., S.B., S.M.G., R.C.-B., and D.C.; methodology, A.F.M., S.B., R.C.-B., and D.C.; software, P.C.K., H.L., A.P., and A.M.; validation, A.F.M., A.P., S.B., S.M.G., and D.C.; formal analysis, P.C.K., S.B., H.L., A.P., and A.M.; investigation, L.M.M., L.M.S.L., A.D., B.B., and H.S.S.; resources, A.F.M., J.J.S., S.B., S.M.G., and D.C.; data curation, A.F.M., S.B., S.M.G., and D.C.; writing-original draft preparation, D.C.; writing-review and editing, P.C.K., A.F.M., S.B., H.L., A.D., S.M.G., R.C.-B., D.G.-B., and D.C.; supervision, A.F.M., S.M.G., S.B., R.C.-B., D.G.-B., and D.C.; project administration, A.F.M., S.M.G., S.B., and D.C.; funding acquisition, A.F.M. and D.C. All authors have read and agreed to the published version of the manuscript.

Funding: This research was funded by the São Paulo State Research Support Foundation (FAFESP, Brazil), the National Health Foundation (FUNASA, Brazil), and the Mato Grosso State Research Support Foundation (FAPEMAT, Brazil), grant number 0839/2006. Additional funding was obtained from the Health Institute Carlos III (ISCIII), Ministry of Economy and Competitiveness (Spain), grant number PI16CIII/00024.

Institutional Review Board Statement: The study was conducted according to the guidelines of the Declaration of Helsinki and approved by the Institutional Review Board of Instituto de Ciências Biomédicas da Universidade de São Paulo (protocol code 14485—CONEP—on 17/06/2008).

Informed Consent Statement: Informed consent was obtained from all subjects involved in the study.

Data Availability Statement: All relevant data are within the article and its additional files. The sequences obtained in this study have been deposited in GenBank under accession numbers MT542718MT542765 ( $g d h)$, MT542766-MT542794 (bg), and MT542795-MT542829 (tpi).

Acknowledgments: S.M. Gennari, A. Marcili, and J. J. Shaw are in receipt of a Research Productivity Fellowship from the National Council for Scientific and Technological Development (CNPq, Brazil).

Conflicts of Interest: The authors declare no conflict of interest. The funders had no role in the design of the study; in the collection, analyses, or interpretation of data; in the writing of the manuscript, or in the decision to publish the results.

\section{References}

1. Einarsson, E.; Ma'Ayeh, S.; Svärd, S.G. An up-date on Giardia and giardiasis. Curr. Opin. Microbiol. 2016, 34, 47-52. [CrossRef]

2. Ankarklev, J.; Hestvik, E.; Lebbad, M.; Lindh, J.; Kaddu-Mulindwa, D.H.; Andersson, J.O.; Tylleskär, T.; Tumwine, J.K.; Svärd, S.G. Common coinfections of Giardia intestinalis and Helicobacter pylori in non-symptomatic Ugandan Children. PLoS Negl. Trop. Dis. 2012, 6, 2-9. [CrossRef]

3. Muadica, A.S.; Balasegaram, S.; Beebeejaun, K.; Köster, P.C.; Bailo, B.; Hernández-De-Mingo, M.; Dashti, A.; DaCal, E.; Saugar, J.M.; Fuentes, I.; et al. Risk associations for intestinal parasites in symptomatic and asymptomatic schoolchildren in central Mozambique. Clin. Microbiol. Infect. 2020. [CrossRef]

4. Reh, L.; Muadica, A.S.; Köster, P.C.; Balasegaram, S.; Verlander, N.Q.; Chércoles, E.R.; Carmena, D. Substantial prevalence of enteroparasites Cryptosporidium spp., Giardia duodenalis and Blastocystis sp. in asymptomatic schoolchildren in Madrid, Spain, November 2017 to June 2018. Eurosurveillance 2019, 24, 1900241. [CrossRef]

5. Kotloff, K.L.; Nataro, J.P.; Blackwelder, W.C.; Nasrin, D.; Farag, T.H.; Panchalingam, S.; Wu, Y.; Sow, O.S.; Sur, D.; Breiman, R.F.; et al. Burden and aetiology of diarrhoeal disease in infants and young children in developing countries (the Global Enteric Multicenter Study, GEMS): A prospective, case-control study. Lancet 2013, 382, 209-222. [CrossRef]

6. Tellevik, M.G.; Moyo, S.J.; Blomberg, B.; Hjøllo, T.; Maselle, S.Y.; Langeland, N.; Hanevik, K. Prevalence of Cryptosporidium parvum/hominis, Entamoeba histolytica and Giardia lamblia among young children with and without Diarrhea in Dar es Salaam, Tanzania. PLoS Negl. Trop. Dis. 2015, 9, e0004125. [CrossRef] [PubMed]

7. $\quad$ Breurec, S.; Vanel, N.; Onambélé, M.; Rafaï, C.; Razakandrainibe, R.; Tondeur, L.; Tricou, V.; Sansonetti, P.J.; Vray, M.; Bata, P.; et al. Etiology and Epidemiology of Diarrhea in Hospitalized Children from Low Income Country: A Matched Case-Control Study in Central African Republic. PLoS Negl. Trop. Dis. 2016, 10, e0004283. [CrossRef] [PubMed] 
8. Dupont, H.L. Giardia: Both a harmless commensal and a devastating pathogen. J. Clin. Investig. 2013, 123, 2352-2354. [CrossRef] [PubMed]

9. Xiao, L.; Feng, Y. Molecular epidemiologic tools for waterborne pathogens Cryptosporidium spp. and Giardia duodenalis. Food Waterborne Parasitol. 2017, 8-9, 14-32. [CrossRef]

10. Certad, G.; Viscogliosi, E.; Chabé, M.; Cacciò, S.M. Pathogenic mechanisms of cryptosporidium and Giardia. Trends Parasitol. 2017, 33, 561-576. [CrossRef] [PubMed]

11. Berkman, D.S.; Lescano, A.G.; Gilman, R.H.; Lopez, S.L.; Black, M.M. Effects of stunting, diarrhoeal disease, and parasitic infection during infancy on cognition in late childhood: A follow-up study. Lancet 2002, 359, 564-571. [CrossRef]

12. Al-Mekhlafi, H.M.; Al-Maktari, M.T.; Jani, R.; Ahmed, A.; Anuar, T.S.; Moktar, N.; Mahdy, M.A.K.; Lim, Y.A.L.; Mahmud, R.; Surin, J. Burden of Giardia duodenalis infection and its adverse effects on growth of schoolchildren in rural Malaysia. PLoS Negl. Trop. Dis. 2013, 7, e2516. [CrossRef] [PubMed]

13. Savioli, L.; Smith, H.; Thompson, A. Giardia and Cryptosporidium join the 'Neglected Diseases Initiative'. Trends Parasitol. 2006, 22, 203-208. [CrossRef]

14. Cacciò, S.M.; Lalle, M.; Svärd, S.G. Host specificity in the Giardia duodenalis species complex. Infect. Genet. Evol. 2018, 66, 335-345. [CrossRef] [PubMed]

15. Ryan, U.; Cacciò, S.M. Zoonotic potential of Giardia. Int. J. Parasitol. 2013, 43, 943-956. [CrossRef] [PubMed]

16. Coelho, C.H.; Durigan, M.; Leal, D.A.G.; Schneider, A.D.B.; Franco, R.M.B.; Singer, S.M. Giardiasis as a neglected disease in Brazil: Systematic review of 20 years of publications. PLoS Negl. Trop. Dis. 2017, 11, e0006005. [CrossRef] [PubMed]

17. Miranda, R.A.; Xavier, F.B.; Menezes, R.C. Intestinal parasitism in a Parakanã indigenous community in southwestern Pará State, Brazil. Cad Saude Publica 1998, 14, 507-511. [CrossRef] [PubMed]

18. Bóia, M.N.; Carvalho-Costa, F.A.; Sodré, F.C.; Porras-Pedroza, B.E.; Faria, E.C.; Magalhães, G.A.; Silva, I.M. Tuberculosis and intestinal parasitism among indigenous people in the Brazilian Amazon region. Rev. Saude Publica 2009, 43, 176-178. [CrossRef] [PubMed]

19. Escobar-Pardo, M.L.; De Godoy, A.P.O.; Machado, R.S.; Rodrigues, D.; Neto, U.F.; Kawakami, E. Prevalence of Helicobacter pylori infection and intestinal parasitosis in children of the Xingu Indian Reservation. J. Pediatr. 2011, 87, 393-398. [CrossRef] [PubMed]

20. Assis, E.M.; Olivieria, R.C.; Moreira, L.E.; Pena, J.L.; Rodrigues, L.C.; Machado-Coelho, G.L. Prevalence of intestinal parasites in the Maxakali indigenous community in Minas Gerais, Brazil, 2009. Cad Saude Publica 2013, 29, 681-690. [CrossRef] [PubMed]

21. Simões Bdos, S.; Machado-Coelho, G.L.; Pena, J.L.; de Freitas, S.N. Environmental conditions and prevalence of parasitic infection in Xukuru-Kariri indigenous people, Caldas, Brazil. Rev. Panam. Salud Publica 2015, 38, 42-48. [PubMed]

22. Neres-Norberg, A.; Guerra-Sanches, F.; Moreira-Norberg, P.R.B.; Madeira-Oliveira, J.T.; Santa-Helena, A.A.; Serra-Freire, N.M. Intestinal parasitism in Terena indigenous people of the Province of Mato Grosso do Sul, Brazil. Rev. Salud Pública 2015, 16, 859-870.

23. Platts-Mills, A.J.; Babji, S.; Bodhidatta, L.; Gratz, J.; Haque, R.; Havt, A.; McCormick, B.J.; McGrath, M.; Olortegui, M.P.; Samie, A.; et al. Pathogen-specific burdens of community diarrhoea in developing countries: A multisite birth cohort study (MAL-ED). Lancet Glob. Health 2015, 3, e564-e575. [CrossRef]

24. Bartelt, L.A.; Platts-Mills, J.A. Giardia: A pathogen or commensal for children in high-prevalence settings? Curr. Opin. Infect. Dis. 2016, 29, 502-507. [CrossRef] [PubMed]

25. GBD 2013 Mortality and Causes of Death Collaborators. Global, regional, and national age-sex specific all-cause and cause-specific mortality for 240 causes of death, 1990-2013: A systematic analysis for the Global Burden of Disease Study 2013. Lancet 2015, 385, 117-171. [CrossRef]

26. Stensvold, C.R. Pinning down the role of common luminal intestinal parasitic protists in human health and disease-status and challenges. Parasitology 2019, 146, 695-701. [CrossRef]

27. Kohli, A.; Bushen, O.Y.; Pinkerton, R.C.; Houpt, E.; Newman, R.D.; Sears, C.L.; Lima, A.A.M.; Guerrant, R.L. Giardia duodenalis assemblage, clinical presentation and markers of intestinal inflammation in Brazilian children. Trans. R. Soc. Trop. Med. Hyg. 2008, 102, 718-725. [CrossRef]

28. Hanevik, K.; Hausken, T.; Morken, M.H.; Strand, E.A.; Mørch, K.; Coll, P.; Helgeland, L.; Langeland, N. Persisting symptoms and duodenal inflammation related to Giardia duodenalis infection. J. Infect. 2007, 55, 524-530. [CrossRef] [PubMed]

29. Donowitz, J.R.; Alam, M.; Kabir, M.; Ma, J.Z.; Nazib, F.; Platts-Mills, J.A.; Bartelt, L.A.; Haque, R.; Petri, W.A. A prospective longitudinal cohort to investigate the effects of early life giardiasis on growth and all cause diarrhea. Clin. Infect. Dis. 2016, 63, 792-797. [CrossRef] [PubMed]

30. Fink, M.Y.; Singer, S.M. The intersection of immune responses, microbiota, and pathogenesis in giardiasis. Trends Parasitol. 2017, 33, 901-913. [CrossRef] [PubMed]

31. Wang, Y.; Gonzalez-Moreno, O.; Roellig, D.M.; Oliver, L.; Huguet, J.; Guo, Y.; Feng, Y.; Xiao, L. Epidemiological distribution of genotypes of Giardia duodenalis in humans in Spain. Parasites Vectors 2019, 12, 1-10. [CrossRef] [PubMed]

32. Sahagún, J.; Clavel, A.; Goñi, P.; Seral, C.; Llorente, M.T.; Castillo, F.J.; Capilla, S.; Arias, A.; Gómez-Lus, R. Correlation between the presence of symptoms and the Giardia duodenalis genotype. Eur. J. Clin. Microbiol. Infect. Dis. 2007, 27, 81-83. [CrossRef]

33. Messa, A., Jr.; Köster, P.C.; Garrine, M.; Gilchrist, C.; Bartelt, L.A.; Nhampossa, T.; Massora, S.; Kotloff, K.; Levine, M.M.; Alonso, P.L.; et al. Molecular diversity of Giardia duodenalis in children under 5 years from the Manhiça district, Southern Mozambique enrolled in a matched case-control study on the aetiology of diarrhoea. PLoS Negl. Trop. Dis. 2021, 15, e0008987. [CrossRef] 
34. Haque, R.; Mondal, D.; Petri, J.W.A.; Karim, A.; Molla, I.H.; Rahim, A.; Faruque, A.S.G.; Ahmad, N.; Kirkpatrick, B.D.; Houpt, E.; et al. Prospective case-control study of the association between common enteric protozoal parasites and diarrhea in Bangladesh. Clin. Infect. Dis. 2009, 48, 1191-1197. [CrossRef]

35. Aydin, A.F.; Besirbellioglu, B.A.; Avci, I.Y.; Tanyuksel, M.; Araz, E.; Pahsa, A. Classification of Giardia duodenalis parasites in Turkey into groups A and B using restriction fragment length polymorphism. Diagn. Microbiol. Infect. Dis. 2004, 50, 147-151. [CrossRef]

36. Homan, W.L.; Mank, T.G. Human giardiasis: Genotype linked differences in clinical symptomatology. Int. J. Parasitol. 2001, 31, 822-826. [CrossRef]

37. Nunes, B.C.; Calegar, D.A.; Pavan, M.G.; Jaeger, L.H.; Monteiro, K.J.L.; Dos Reis, E.R.C.; Lima, M.M.; Neves Bóia, M.; CarvalhoCosta, F.A. Genetic diversity of Giardia duodenalis circulating in three Brazilian biomes. Infect. Genet. Evol. 2018, 59, 107-112. [CrossRef]

38. De Lucio, A.; Amor-Aramendía, A.; Bailo, B.; Saugar, J.M.; Anegagrie, M.; Arroyo, A.; López-Quintana, B.; Zewdie, D.; Ayehubizu, Z.; Yizengaw, E.; et al. Prevalence and genetic diversity of Giardia duodenalis and Cryptosporidium spp. among school children in a Rural Area of the Amhara Region, North-West Ethiopia. PLoS ONE 2016, 11, e0159992. [CrossRef]

39. Dacal, E.; Saugar, J.M.; De Lucio, A.; Hernández-De-Mingo, M.; Robinson, E.; Köster, P.C.; Aznar-Ruiz-De-Alegría, M.L.; Espasa, M.; Ninda, A.; Gandasegui, J.; et al. Prevalence and molecular characterization of Strongyloides stercoralis, Giardia duodenalis, Cryptosporidium spp., and Blastocystis spp. isolates in school children in Cubal, Western Angola. Parasites Vectors 2018, 11, 1-18. [CrossRef] [PubMed]

40. Oliveira-Arbex, A.P.; David, E.B.; Oliveira-Sequeira, T.C.G.; Bittencourt, G.N.; Guimaraes, S.T.D.L. Genotyping of Giardia duodenalis isolates in asymptomatic children attending daycare centre: Evidence of high risk for anthroponotic transmission. Epidemiol. Infect. 2015, 144, 1418-1428. [CrossRef] [PubMed]

41. Seguí, R.; Muñoz-Antoli, C.; Klisiowicz, D.R.; Oishi, C.Y.; Köster, P.C.; De Lucio, A.; Hernández-De-Mingo, M.; Puente, P.; Toledo, R.; Esteban, J.G.; et al. Prevalence of intestinal parasites, with emphasis on the molecular epidemiology of Giardia duodenalis and Blastocystis sp., in the Paranaguá Bay, Brazil: A community survey. Parasites Vectors 2018, 11, 1-19. [CrossRef]

42. Prabhu, A.; Morrison, H.G.; Martinez, C.R.; Adam, R.D. Characterisation of the subtelomeric regions of Giardia lamblia genome isolate WBC6. Int. J. Parasitol. 2007, 37, 503-513. [CrossRef] [PubMed]

43. Teodorovic, S.; Braverman, J.M.; Elmendorf, H.G. Unusually Low Levels of Genetic Variation among Giardia lamblia Isolates. Eukaryot. Cell 2007, 6, 1421-1430. [CrossRef]

44. Ankarklev, J.; Svärd, S.G.; Lebbad, M. Allelic sequence heterozygosity in single Giardia parasites. BMC Microbiol. 2012, 12, 65. [CrossRef]

45. Siripattanapipong, S.; Leelayoova, S.; Mungthin, M.; Thompson, R.A.; Boontanom, P.; Saksirisampant, W.; Tan-Ariya, P. Clonal diversity of the glutamate dehydrogenase gene in Giardia duodenalis from Thai Isolates: Evidence of genetic exchange or mixed infections? BMC Microbiol. 2011, 11, 206. [CrossRef]

46. Seabolt, M.H.; Konstantinidis, K.T.; Roellig, D.M. Hidden diversity within common protozoan parasites revealed by a novel genomotyping scheme. Appl. Environ. Microbiol. 2021. [CrossRef] [PubMed]

47. Ankarklev, J.; Lebbad, M.; Einarsson, E.; Franzén, O.; Ahola, H.; Troell, K.; Svärd, S.G. A novel high-resolution multilocus sequence typing of Giardia intestinalis Assemblage A isolates reveals zoonotic transmission, clonal outbreaks and recombination. Infect. Genet. Evol. 2018, 60, 7-16. [CrossRef]

48. Instituto Brasileiro de Geografia e Estatística. Indígenas. Available online: https://indigenas.ibge.gov.br/ (accessed on 4 November 2020).

49. Fundação Nacional do Índio. Índios do Brasil. Available online: http:/ / www.funai.gov.br/funai/ (accessed on 4 November 2020).

50. Instituto Sócio-Ambiental. Povos Indígenas no Brasil. Available online: http://www.socioambiental.org/ (accessed on 4 November 2020).

51. Sheather, A.L. The detection of intestinal protozoa and mange parasites by a flotation technique. J Comp. Pathol. 1936, 36, 266-275. [CrossRef]

52. Hoffman, W.A.; Pons, J.A.; Janer, J.L. The sedimentation concentration method in Schistosomiasis mansoni. J. Public Health Trop. Med. 1934, 9, 283-291.

53. Allen, A.V.; Ridley, D.S. Further observations on the formol-ether concentration technique for faecal parasites. J. Clin. Pathol. 1970, 23, 545-546. [CrossRef] [PubMed]

54. Faust, E.C.; D'Antoni, J.S.; Odom, V.; Miller, M.J.; Peres, C.; Sawitz, W.; Thomen, L.F.; Tobie, J.; Walker, J.H. A critical study of clinical laboratory technics for the diagnosis of protozoan cysts and helminth eggs in feces 1. Am. J. Trop. Med. Hyg. 1938, 18, 169-183. [CrossRef]

55. Weiss, J.B. PCR detection of Giardia lamblia. In Diagnostic Molecular Microbiology: Principles and Applications; Persing, D.H., Smith, T.F., Tenover, F.C., White, T.J., Eds.; American Society for Microbiology: Washington, WA, USA, 1993; pp. 480-485.

56. Verweij, J.J.; Schinkel, J.; Laeijendecker, D.; Van Rooyen, M.A.A.; Van Lieshout, L.; Polderman, A.M. Real-time PCR for the detection of Giardia lamblia. Mol. Cell. Probes 2003, 17, 223-225. [CrossRef]

57. Read, C.M.; Monis, P.T.; Thompson, R.C. Discrimination of all genotypes of Giardia duodenalis at the glutamate dehydrogenase locus using PCR-RFLP. Infect. Genet. Evol. 2004, 4, 125-130. [CrossRef] 
58. Lalle, M.; Pozio, E.; Capelli, G.; Bruschi, F.; Crotti, D.; Cacciò, S.M. Genetic heterogeneity at the beta-giardin locus among human and animal isolates of Giardia duodenalis and identification of potentially zoonotic subgenotypes. Int. J. Parasitol. 2005, 35, 207-213. [CrossRef]

59. Sulaiman, I.M.; Fayer, R.; Bern, C.; Gilman, R.H.; Trout, J.M.; Schantz, P.M.; Das, P.; Al, I.M.S.E.; Xiao, L. Triosephosphate isomerase gene characterization and potential zoonotic transmission of Giardia duodenalis. Emerg. Infect. Dis. 2003, 9, 1444-1452. [CrossRef] [PubMed]

60. Tamura, K.; Stecher, G.; Peterson, D.; Filipski, A.; Kumar, S. MEGA6: Molecular evolutionary genetics analysis version 6.0. Mol. Biol. Evol. 2013, 30, 2725-2729. [CrossRef]

61. Thompson, J.D.; Gibson, T.J.; Plewniak, F.; Jeanmougin, F.; Higgins, D.G. The CLUSTAL_X windows interface: Flexible strategies for multiple sequence alignment aided by quality analysis tools. Nucleic Acids Res. 1997, 25, 4876-4882. [CrossRef]

62. Nicholas, K.B.; Nicholas, H.B., Jr. GeneDoc: A Tool for Editing and Annotating Multiple Sequence Alignments. (Distributed by the Author). 1997. Available online: https:/ / www.scienceopen.com/document?vid=c8a87cd1-255f-4129-802b-d2382bb0fb37 (accessed on 11 February 2021).

63. Swofford, D.L. PAUP* Version 4.0 b10. Phylogenetic Analysis Using Parsimony (* and Other Methods); Sinauer: Sunderland, UK, 2002.

64. Huelsenbeck, J.P.; Ronquist, F. MRBAYES: Bayesian inference of phylogenetic trees. Bioinformatics 2001, 17, 754-755. [CrossRef]

65. Pacheco, F.T.; Carvalho, S.S.; Teixeira, M.C.; Cardoso, L.S.; Andrade, L.S.; Das Chagas, G.M.; Gomes, D.C.; Mercês, C.F.; Rocha, F.C.; Silva, L.K.; et al. Immune response markers in sera of children infected with Giardia duodenalis AI and AII subassemblages. Immunobiology 2019, 224, 595-603. [CrossRef] [PubMed]

66. Santos, C.K.S.; Grama, D.F.; Limongi, J.E.; Costa, F.C.; Couto, T.R.; Soares, R.M.; Mundim, M.J.S.; Cury, M.C. Epidemiological, parasitological and molecular aspects of Giardia duodenalis infection in children attending public daycare centers in southeastern Brazil. Trans. R. Soc. Trop. Med. Hyg. 2012, 106, 473-479. [CrossRef]

67. Scalia, L.A.; Fava, N.M.; Soares, R.M.; Limongi, J.E.; da Cunha, M.J.; Pena, I.F.; Kalapothakis, E.; Cury, M.C. Multilocus genotyping of Giardia duodenalis in Brazilian children. Trans. R. Soc. Trop. Med. Hyg. 2016, 110, 343-349. [CrossRef] [PubMed]

68. Gomes, K.B.; Fernandes, A.P.; Menezes, A.; Júnior, R.A.; Silva, E.F.; Rocha, M.O. Giardia duodenalis: Genotypic comparison between a human and a canine isolates. Rev. Soc. Bras. Med. Trop. 2011, 44, 508-510. [CrossRef]

69. Uda-Shimoda, C.F.; Colli, C.M.; Pavanelli, M.F.; Falavigna-Guilherme, A.L.; Gomes, M.L. Simplified protocol for DNA extraction and amplification of 2 molecular markers to detect and type Giardia duodenalis. Diagn. Microbiol. Infect. Dis. 2014, 78, 53-58. [CrossRef] [PubMed]

70. Colli, C.M.; Bezagio, R.C.; Nishi, L.; Bignotto, T.S.; Ferreira, É.C.; Falavigna-Guilherme, A.L.; Gomes, M.L. Identical assemblage of Giardia duodenalis in humans, animals and vegetables in an urban area in Southern Brazil indicates a relationship among them. PLOS ONE 2015, 10, e0118065. [CrossRef]

71. Colli, C.M.; Bezagio, R.C.; Nishi, L.; Ferreira, É.C.; Falavigna-Guilherme, A.L.; Gomes, M.L. Food handlers as a link in the chain of transmission of Giardia duodenalis and other protozoa in public schools in southern Brazil: Table 1. Trans. R. Soc. Trop. Med. Hyg. 2015, 109, 601-603. [CrossRef] [PubMed]

72. Volotão, A.C.; Costa-Macedo, L.M.; Haddad, F.S.; Brandão, A.; Peralta, J.M.; Fernandes, O. Genotyping of Giardia duodenalis from human and animal samples from Brazil using beta-giardin gene: A phylogenetic analysis. Acta Trop. 2007, 102, 10-19. [CrossRef]

73. Faria, C.P.; Zanini, G.M.; Dias, G.S.; Da Silva, S.; Sousa, M.D.C. Molecular Characterization of Giardia lamblia: First Report of Assemblage B in Human Isolates from Rio de Janeiro (Brazil). PLoS ONE 2016, 11, e0160762. [CrossRef]

74. Faria, C.P.; Zanini, G.M.; Dias, G.S.; Sousa, M.D.C. Associations of Giardia lamblia assemblages with HIV infections and symptomatology: HIV virus and assemblage B were they born to each other? Acta Trop. 2017, 172, 80-85. [CrossRef]

75. Faria, C.P.; Zanini, G.M.; Dias, G.S.; Da Silva, S.; Sousa, M.D.C. New multilocus genotypes of Giardia lamblia human isolates. Infect. Genet. Evol. 2017, 54, 128-137. [CrossRef] [PubMed]

76. Cascais-Figueiredo, T.; Austriaco-Teixeira, P.; Fantinatti, M.; Silva-Freitas, M.L.; Santos-Oliveira, J.R.; Coelho, C.H.; Singer, S.M.; Da-Cruz, A.M. Giardiasis alters intestinal fatty acid binding protein (I-FABP) And plasma cytokines levels in children in Brazil. Pathogens 2019, 9, 7. [CrossRef] [PubMed]

77. Fantinatti, M.; Bello, A.R.; Fernandes, O.; Da-Cruz, A.M. Identification of Giardia lamblia assemblage E in humans points to a new anthropozoonotic cycle. J. Infect. Dis. 2016, 214, 1256-1259. [CrossRef]

78. De Quadros, R.M.; Weiss, P.H.E.; Marques, S.M.T.; Miletti, L.C. Potential cross-contamination of similar giardia duodenalis assemblage in children and pet dogs in Southern Brazil, as determined by PCR-RFLP. Rev. Inst. Med. Trop. São Paulo 2016, 58. [CrossRef]

79. De Godoy, E.A.M.; Junior, J.E.S.; Belloto, M.V.T.; De Moraes, M.V.P.; Cassiano, G.C.; Volotao, A.C.C.; Luvizotto, M.C.R.; Carareto, C.M.A.; Silva, M.C.D.M.; Machado, R.L.D. Molecular investigation of zoonotic genotypes of Giardia intestinalis isolates in humans, dogs and cats, sheep, goats and cattle in Araçatuba (São Paulo State, Brazil) by the analysis of ß-giardin gene fragments. Microbiol. Res. 2013, 4, e6. [CrossRef]

80. Coradi, S.; David, E.; Oliveira-Sequeira, T.; Ribolla, P.; Carvalho, T.; Guimarães, S. Genotyping of Brazilian Giardia duodenalis human axenic isolates. J. Venom. Anim. Toxins Incl. Trop. Dis. 2011, 17, 353-357. [CrossRef] 
81. Durigan, M.; Abreu, A.G.; Zucchi, M.I.; Franco, R.M.B.; De Souza, A.P. Genetic diversity of Giardia duodenalis: Multilocus genotyping reveals zoonotic potential between clinical and environmental sources in a metropolitan region of Brazil. PLoS ONE 2014, 9, e115489. [CrossRef] [PubMed]

82. Corrêa, C.R.T.; Oliveira-Arbex, A.P.; David, É.B.; Guimarães, S. Genetic analysis of Giardia duodenalis isolates from children of low-income families living in an economically successful region in Southeastern Brazil. Rev. Inst. Med. Trop. Sao Paulo 2020, 62, e20. [CrossRef]

83. Volotão, A.C.; Ramos, N.M.; Fantinatti, M.; Moraes, M.V.; Netto, H.A.; Storti-Melo, L.M.; Godoy, E.A.; Rossit, A.R.; Fernandes, O.; Machado, R.L. Giardiasis as zoonosis: Between proof of principle and paradigm in the Northwestern region of São Paulo State, Brazil. Braz. J. Infect. Dis. 2011, 15, 382-383. [CrossRef] [PubMed]

84. David, É.B.; Guimarães, S.; de Oliveira, A.P.; de Oliveira-Sequeira, T.C.; Nogueira Bittencourt, G.; Moraes Nardi, A.R.; Martins Ribolla, P.E.; Bueno Franco, R.M.; Branco, N.; Tosini, F.; et al. Molecular characterization of intestinal protozoa in two poor communities in the State of São Paulo, Brazil. Parasit Vectors 2015, 8, 103. [CrossRef]

85. Souza, S.L.; Gennari, S.M.; Richtzenhain, L.J.; Pena, H.F.; Funada, M.R.; Cortez, A.; Gregori, F.; Soares, R.M. Molecular identification of Giardia duodenalis isolates from humans, dogs, cats and cattle from the state of São Paulo, Brazil, by sequence analysis of fragments of glutamate dehydrogenase (gdh) coding gene. Veter. Parasitol. 2007, 149, 258-264. [CrossRef] [PubMed]

86. Da Cunha, M.J.R.; Cury, M.C.; Santín, M. Molecular identification of Enterocytozoon bieneusi, Cryptosporidium, and Giardia in Brazilian captive birds. Parasitol. Res. 2016, 116, 487-493. [CrossRef]

87. Fava, N.M.N.; Soares, R.M.; Scalia, L.A.M.; Kalapothakis, E.; Pena, I.F.; Vieira, C.U.; Faria, E.S.M.; Cunha, M.J.; Couto, T.R.; Cury, M.C. Performance of glutamate dehydrogenase and triose phosphate isomerase genes in the analysis of genotypic variability of isolates of Giardia duodenalis from livestocks. BioMed Res. Int. 2013, 2013, 1-9. [CrossRef]

88. Fava, N.M.; Soares, R.M.; Scalia, L.A.; Da Cunha, M.J.R.; Faria, E.S.; Cury, M.C. Molecular typing of canine Giardia duodenalis isolates from Minas Gerais, Brazil. Exp. Parasitol. 2016, 161, 1-5. [CrossRef] [PubMed]

89. Meireles, P.; Montiani-Ferreira, F.; Thomaz-Soccol, V. Survey of giardiosis in household and shelter dogs from metropolitan areas of Curitiba, Paraná state, Southern Brazil. Veter. Parasitol. 2008, 152, 242-248. [CrossRef]

90. Uchôa, F.F.D.M.; Sudré, A.P.; Campos, S.D.E.; Almosny, N.R.P. Assessment of the diagnostic performance of four methods for the detection of Giardia duodenalis in fecal samples from human, canine and feline carriers. J. Microbiol. Methods 2018, 145, 73-78. [CrossRef]

91. Fantinatti, M.; Caseca, A.C.; Bello, A.R.; Fernandes, O.; Da-Cruz, A.M.; Filho, O.F.D.S. The presence of Giardia lamblia assemblage A in dogs suggests an anthropozoonotic cycle of the parasite in Rio de Janeiro, Brazil. Infect. Genet. Evol. 2018, 65, 265-269. [CrossRef]

92. Volotão, A.; Júnior, J.S.; Grassini, C.; Peralta, J.; Fernandes, O. Genotyping of Giardia duodenalis from Southern Brown Howler Monkeys (Alouatta clamitans) from Brazil. Veter. Parasitol. 2008, 158, 133-137. [CrossRef] [PubMed]

93. Soares, R.M.; De Souza, S.L.P.; Silveira, L.H.; Funada, M.R.; Richtzenhain, L.J.; Gennari, S.M. Genotyping of potentially zoonotic Giardia duodenalis from exotic and wild animals kept in captivity in Brazil. Veter. Parasitol. 2011, 180, 344-348. [CrossRef] [PubMed]

94. De Aquino, M.C.C.; Harvey, T.V.; Inácio, S.V.; Nagata, W.B.; Ferrari, E.D.; Oliveira, B.C.M.; Albuquerque, G.R.; Widmer, G.; Meireles, M.V.; Bresciani, K.D.S. First description of Giardia duodenalis in buffalo calves (Bubalus bubalis) in southwest region of São Paulo State, Brazil. Food Waterborne Parasitol. 2019, 16, e00062. [CrossRef] [PubMed]

95. David, E.B.; Patti, M.; Coradi, S.T.; Oliveira-Sequeira, T.C.G.; Ribolla, P.E.M.; Guimaraes, S. Molecular typing of Giardia duodenalis isolates from nonhuman primates housed in a Brazilian zoo. Rev. Inst. Med. Trop. São Paulo 2014, 56, 49-54. [CrossRef] [PubMed]

96. e Silva, F.M.P.; Monobe, M.M.; Lopes, R.S.; Araujo, J.P. Molecular characterization of Giardia duodenalis in dogs from Brazil. Parasitol. Res. 2012, 110, 325-334. [CrossRef] [PubMed]

97. Silva, F.M.P.; Lopes, R.S.; Araújo, J.P.; Paze, F.S.M. Genetic characterisation of Giardia duodenalis in dairy cattle in Brazil. Folia Parasitol. 2012, 59, 15-20. [CrossRef]

98. Silva, F.P.E.; Lopes, R.; Bresciani, K.D.S.; Amarante, A.; Araujo, J. High occurrence of Cryptosporidium ubiquitum and Giardia duodenalis genotype E in sheep from Brazil. Acta Parasitol. 2014, 59, 193-196. [CrossRef]

99. Ferreira, F.P.; Caldart, E.T.; Freire, R.L.; Mitsuka-Breganó, R.; De Freitas, F.M.; Miura, A.C.; Mareze, M.; Martins, F.D.C.; Urbano, M.R.; Seifert, A.L.; et al. The effect of water source and soil supplementation on parasite contamination in organic vegetable gardens. Rev. Bras. Parasitol. Vet. 2018, 27, 327-337. [CrossRef]

100. Almeida, J.C.; Martins, F.D.C.; Neto, J.M.F.; Dos Santos, M.M.; Garcia, J.L.; Navarro, I.T.; Kuroda, E.K.; Freire, R.L. Occurrence of Cryptosporidium spp. and Giardia spp. in a public water-treatment system, Paraná, Southern Brazil. Rev. Bras. Parasitol. Vet. 2015, 24, 303-308. [CrossRef]

101. Ladeia, W.A.; Martins, F.D.C.; e Silva, C.F.R.; Freire, R.L. Molecular surveillance of Cryptosporidium and Giardia duodenalis in sludge and spent filter backwash water of a water treatment plant. J. Water Health 2018, 16, 857-860. [CrossRef] [PubMed]

102. Souza, D.S.M.; Ramos, A.P.D.; Nunes, F.F.; Moresco, V.; Taniguchi, S.; Leal, D.A.G.; Sasaki, S.T.; Bícego, M.C.; Montone, R.C.; Durigan, M.; et al. Evaluation of tropical water sources and mollusks in southern Brazil using microbiological, biochemical, and chemical parameters. Ecotoxicol. Environ. Saf. 2012, 76, 153-161. [CrossRef] 
103. De Araújo, R.S.; Aguiar, B.; Dropa, M.; Razzolini, M.T.P.; Sato, M.I.Z.; Lauretto, M.D.S.; Galvani, A.T.; Padula, J.A.; Matté, G.R.; Matté, M.H. Detection and molecular characterization of Cryptosporidium species and Giardia assemblages in two watersheds in the metropolitan region of São Paulo, Brazil. Environ. Sci. Pollut. Res. 2018, 25, 15191-15203. [CrossRef]

104. Ulloa-Stanojlović, F.M.; Aguiar, B.; Jara, L.M.; Sato, M.I.Z.; Guerrero, J.A.; Hachich, E.; Matté, G.R.; Dropa, M.; Matté, M.H.; De Araújo, R.S. Occurrence of Giardia intestinalis and Cryptosporidium sp. in wastewater samples from São Paulo State, Brazil, and Lima, Peru. Environ. Sci. Pollut. Res. 2016, 23, 22197-22205. [CrossRef] [PubMed]

105. Yamashiro, S.; Foco, M.L.R.; Pineda, C.O.; José, J.; Nour, E.A.A.; Siqueira-Castro, I.C.V.; Franco, R.M.B. Giardia spp. and Cryptosporidium spp. removal efficiency of a combined fixed-film system treating domestic wastewater receiving hospital effluent. Environ. Sci. Pollut. Res. 2019, 26, 22756-22771. [CrossRef]

106. Leal, D.A.G.; Souza, D.S.M.; Caumo, K.S.; Fongaro, G.; Panatieri, L.F.; Durigan, M.; Rott, M.B.; Barardi, C.R.M.; Franco, R.M.B. Genotypic characterization and assessment of infectivity of human waterborne pathogens recovered from oysters and estuarine waters in Brazil. Water Res. 2018, 137, 273-280. [CrossRef]

107. Fernandes, L.N.; De Souza, P.P.; De Araújo, R.S.; Razzolini, M.T.P.; Soares, R.M.; Sato, M.I.Z.; Hachich, E.M.; Cutolo, S.A.; Matté, G.R.; Matté, M.H. Detection of assemblages A and B of Giardia duodenalis in water and sewage from São Paulo state, Brazil. J. Water Health 2011, 9, 361-367. [CrossRef] [PubMed]

108. Tiyo, R.; De Souza, C.Z.; Piovesani, A.F.A.; Tiyo, B.T.; Colli, C.M.; Marchioro, A.A.; Gomes, M.L.; Falavigna-Guilherme, A.L. Predominance of Giardia duodenalis assemblage aii in fresh leafy vegetables from a market in Southern Brazil. J. Food Prot. 2016, 79, 1036-1039. [CrossRef] [PubMed]

109. Rafael, K.; Marchioro, A.A.; Colli, C.M.; Tiyo, B.T.; Evangelista, F.F.; Bezagio, R.C.; Falavigna-Guilherme, A.L. Genotyping of Giardia duodenalis in vegetables cultivated with organic and chemical fertilizer from street markets and community vegetable gardens in a region of Southern Brazil. Trans. R. Soc. Trop. Med. Hyg. 2017, 111, 540-545. [CrossRef] [PubMed] 